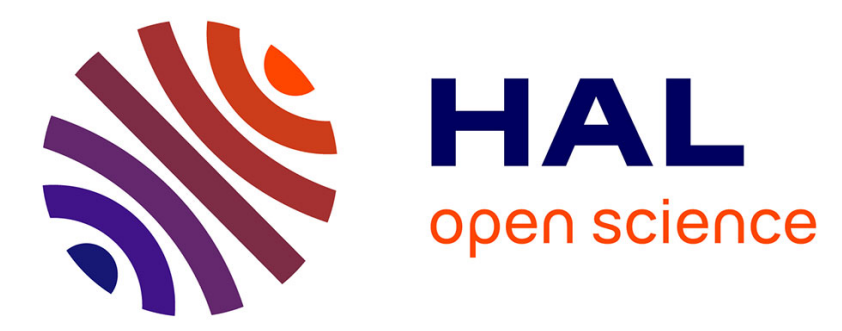

\title{
Instabilities driven by ion shell distributions observed by Cluster in the midaltitude plasma sheet boundary layer.
}

\author{
M. Ashour-Abdalla, J.N. Leboeuf, D. Schriver, J.-M. Bosqued, N.
}

Cornilleau-Wehrlin, V. Sotnikov, A. Marchaudon, A. N. Fazakerley

\section{- To cite this version:}

M. Ashour-Abdalla, J.N. Leboeuf, D. Schriver, J.-M. Bosqued, N. Cornilleau-Wehrlin, et al.. Instabilities driven by ion shell distributions observed by Cluster in the midaltitude plasma sheet boundary layer.. Journal of Geophysical Research Space Physics, 2006, 111 (A10), pp.A10223. 10.1029/2005JA011490 . hal-00153513

\section{HAL Id: hal-00153513 https://hal.science/hal-00153513}

Submitted on 13 Feb 2016

HAL is a multi-disciplinary open access archive for the deposit and dissemination of scientific research documents, whether they are published or not. The documents may come from teaching and research institutions in France or abroad, or from public or private research centers.
L'archive ouverte pluridisciplinaire HAL, est destinée au dépôt et à la diffusion de documents scientifiques de niveau recherche, publiés ou non, émanant des établissements d'enseignement et de recherche français ou étrangers, des laboratoires publics ou privés. 


\title{
Instabilities driven by ion shell distributions observed by Cluster in the midaltitude plasma sheet boundary layer
}

\author{
M. Ashour-Abdalla, ${ }^{1,2}$ J. N. Leboeuf, ${ }^{1}$ D. Schriver, ${ }^{2}$ J.-M. Bosqued, ${ }^{3}$ \\ N. Cornilleau-Wehrlin, ${ }^{4}$ V. Sotnikov, ${ }^{5}$ A. Marchaudon, ${ }^{6}$ and A. N. Fazakerley ${ }^{6}$ \\ Received 21 October 2005; revised 9 February 2006; accepted 6 June 2006; published 24 October 2006.
}

[1] Cluster observations in the near-Earth plasma sheet boundary layer (PSBL) region have shown the presence of ion shell distributions related to velocity-dispersed ion structures (VDIS) coincident with electrostatic emissions. We have examined ion shell instabilities in the presence of a cold ion and electron background using linear theory and particle in cell simulations. Linear theory shows that the shell instability is only excited when a cold ion background is present, generating a broad range of ion cyclotron harmonics. Numerical simulations confirm that ion Bernstein modes are preferentially excited transverse to the ambient magnetic field, along with a lower level of wave power at oblique angles. The background ions are heated primarily in the transverse direction because of a linear nonstochastic ion cyclotron heating mechanism, and overall saturation of the instability occurs because of thermalization of the shell combined with heating of the background ions and electrons. Comparison with Cluster observations shows that observed electrostatic waves with a spectrum from a few $\mathrm{Hz}$ to several hundred $\mathrm{Hz}$ is in good agreement with that expected from the shell instability. The cold background ions are observed to have a temperature anisotropy with $T_{\perp}>T_{\|}$, while electrons are observed to have $T_{\|}>T_{\perp}$, also in qualitative agreement with the shell instability. The wave-particle effects due to the shell instability in the near-Earth PSBL could have important consequences for auroral potential structure at lower altitudes and may cause the gaps in VDIS structure leading to beamlets.

Citation: Ashour-Abdalla, M., J. N. Leboeuf, D. Schriver, J.-M. Bosqued, N. Cornilleau-Wehrlin, V. Sotnikov, A. Marchaudon, and A. N. Fazakerley (2006), Instabilities driven by ion shell distributions observed by Cluster in the midaltitude plasma sheet boundary layer, J. Geophys. Res., 111, A10223, doi:10.1029/2005JA011490.

\section{Introduction}

[2] The Earth's plasma sheet boundary layer (PSBL), which is roughly located on the nightside closed field line region between about $65^{\circ}$ and $75^{\circ}$ invariant latitude, is an important area of transport that magnetically links the lower-altitude auroral zone region and the deep magnetotail plasma sheet source region [Eastman et al., 1984]. Warm plasma accelerated in the far magnetotail streams earthward in the PSBL in the form of energetic $(>10 \mathrm{keV})$ ion beams [Hones et al., 1972; Frank et al., 1976], whereas cooler plasma of ionospheric origin flows tailward from the auroral

\footnotetext{
${ }^{1}$ Department of Physics, University of California, Los Angeles, California, USA.

${ }^{2}$ Institute of Geophysics and Planetary Physics, University of California, Los Angeles, California, USA.

${ }^{3}$ Centre Etude Spatiale des Rayonnements, Centre National de la Recherche Scientifique, Toulouse, France.

${ }^{4}$ Centre d'Etude des Environnements Terrestre et Planétaires, Institut Pierre-Simon Laplace, Velizy, France.

${ }^{5}$ Department of Physics, University of Nevada, Reno, Nevada, USA.

${ }^{6}$ Mullard Space Science Laboratory, University College London, Dorking, UK.
}

Copyright 2006 by the American Geophysical Union. 0148-0227/06/2005JA011490 zone, and these different plasma populations meet in the near-Earth extension of the PSBL at about 4 to $8 \mathrm{R}_{\mathrm{E}}$ from the Earth. The high-energy PSBL ion beams most likely originate as nonadiabatic Speiser ions in the deeper magnetotail near the weak field region of the magnetotail midplane [Lyons and Speiser, 1982; Ashour-Abdalla et al., 1991, 1993]. As the accelerated PSBL ions travel earthward they acquire an enhanced perpendicular velocity and are observed as "kidney bean" shaped distributions [DeCoster and Frank, 1979; Forbes et al., 1981], which is most likely due to a combination of adiabatic mirror heating in the increasing magnetic field, nonadiabatic acceleration that occurred during energization in the magnetotail midplane and/or pitch angle scattering due to wave-particle interactions. In the near-Earth PSBL, due to the nonadiabatic acceleration and time-of-flight effects, the high-energy ions take the form of velocity dispersed ion structures (VDIS), which are characterized by ions that have highest energies at higher latitudes within the closed field line region, and a decreasing energy with decreasing latitude [Bosqued, 1987; Zelenyi et al., 1990]. It has been shown recently that in the high-altitude auroral zone where the terrestrial magnetic field is strong and mirror reflection occurs, the ion distribution functions within the VDIS 
appear as velocity shells that have a drift velocity both parallel and perpendicular to the ambient magnetic field [Janhunen et al., 2003a; Olsson et al., 2004].

[3] Plasma wave activity is commonly observed in the PSBL, including broadband electrostatic noise (BEN), which are intense waves ranging in frequency from below the ion plasma frequency up to the electron plasma frequency [Scarf et al., 1974; Gurnett et al., 1976], spiky dc electric fields [Cattell et al., 1986] and kinetic Alfvén waves [Wygant et al., 2000]. A detailed analysis of the BEN spectrum in the PSBL using Geotail satellite data showed that at least the higher-frequency portion of the spectrum is in the form of electrostatic solitary waves (ESW) [Matsumoto et al., 1994]. In general enhanced wave activity is expected in the PSBL since it is a region of mixed, anisotropic plasma distributions that can be unstable to various waves. For example, it was shown that ion beams in the PSBL can be unstable to BEN-like spectra [Grabbe and Eastman, 1984; Ashour-Abdalla and Okuda, 1986; Schriver and Ashour-Abdalla, 1987] and if a cold electron background is present frequencies up to the electron plasma frequency can be driven unstable, resulting in cold electron heating [Schriver and Ashour-Abdalla, 1990]. Similarly it has been shown that ESW can be driven by electron beams in the PSBL [Omura et al., 1996; Kojima et al., 1999]. It is likely that the entire BEN spectrum is a superposition of modes formed by ion beam or anisotropy instabilities generating the lower-frequency end of the spectrum near the ion cyclotron and ion plasma frequencies and the higher frequencies up to the electron plasma frequency are electron anisotropy driven ESW [Grabbe, 2002]. These studies have shown that the plasma waves generated by the PSBL beams in turn modify the local distributions via waveparticle interactions, affecting plasma transport.

[4] In this paper the focus is on ion driven instabilities in the PSBL. The study of ion beam instabilities in the PSBL has been carried out primarily for the deeper magnetotail $\left(\geq 20 R_{E}\right)$, where the ions are more "beam-like", in the sense that the distribution function is considered as a fieldaligned drifting Maxwellian [e.g., Grabbe and Eastman, 1984; Schriver and Ashour-Abdalla, 1987, 1990]. Beams with enhanced perpendicular thermal velocity such that the beam's perpendicular temperature is greater than the parallel temperature $\left(T_{\perp}>T_{\|}\right)$have also been considered [Nishikawa et al., 1988]. With the recent observations that the ion distributions are in the form of velocity shells in the nearEarth PSBL, the stability of such distributions, as well as the effects of ion shell instabilities on the plasma have also been examined [Janhunen et al., 2003a]. A shell distribution is defined as a distribution function in which the particles are isotropically distributed in velocity space on a sphere with (velocity) radius $v_{s}$. This is compared to a ring distribution whereby the particles have a perpendicular drift velocity, but the parallel drift velocity is zero. Although a shell distribution by itself can be unstable to cyclotron (Bernstein) waves [Tataronis and Crawford, 1970a, 1970b], it was shown that if a cold background plasma is included with the shell, the growth rates are enhanced [Sentman et al., 1986, 1987]. This previous work considered electron shells, but a similar process occurs for ions such that ion shells can also be unstable [Freund and Wu, 1988; Janhunen et al., 2003a; Shevchenko et al., 2004]. For conditions in the high-altitude auroral zone, near-Earth PSBL, it was shown that an ion shell in the presence of a cold ion background was unstable to ion cyclotron waves, which heated the cold background ions and electrons [Janhunen et al., 2003a]. Janhunen et al. [2003a] theorized that the parallel electron heating due to the ion shell instabilities had important ramifications for auroral potential structure at altitudes below where the shells were observed.

[5] To further understand the properties and consequences of ion shell distributions, we have examined recent Cluster wave and particle data during traversals of the nearEarth PSBL, shedding new insight on the processes occurring there. Energetic ion shell distributions are clearly observed, along with cold background ions of ionospheric origin. Coincident with the shell distributions and the cold background plasma are intense broadband electrostatic waves from a few $\mathrm{Hz}$ up to several hundred $\mathrm{Hz}$. At times, electromagnetic emissions with about the same frequencies as the electrostatic waves are also observed. The cold background ions are observed to have a temperature anisotropy with $T_{\perp}>T_{\|}$. A linear theory and simulation study with parameters based on observations shows that the ion shell distribution in the presence of a cold background can excite a broadband series of cyclotron harmonic waves from just above the ion gyrofrequency $\left(f_{\mathrm{ci}}\right)$ up to $\sim 25 f_{\mathrm{ci}}$, explaining the broadband nature of the observed waves. The ion background is heated preferentially in the perpendicular direction and the instability tends to thermalize the shell in velocity space to reduce the free energy. Also as a result of the shell instability, background electrons are heated in the parallel direction forming a distribution with $T_{\|}>T_{\perp}$. The linear theory and simulation results for the shell instability are consistent with ion and electron data, as well as wave data from the Cluster spacecraft.

[6] The paper is organized as follows. In the next section, wave and particle data from the Cluster satellites when they traversed the near-Earth PSBL will be presented. In section 3 the linear theory for a shell distribution is considered for the observed parameters. In section 4 the simulation model to be used to study the ion shell distributions is described and in section 5 the simulation results are presented. The paper concludes in section 6 with a summary of the results and the ramifications for the auroral zone, near-Earth PSBL and the magnetotail.

\section{Experimental Observations}

[7] The observational data relevant to this study was made by the Cluster II satellites when they were in the Earth's magnetotail PSBL region at midaltitude $\left(\sim 4 R_{E}\right)$. The Cluster II satellites were launched in 2000 and placed on an inertial polar orbit of $4 \times 19.6 \mathrm{R}_{\mathrm{E}}$; they consist of 4 identical satellites orbiting the Earth in unison. The separation distance between the 4 satellites has been varied during the mission lifetime from a few hundred to tens of thousands of kilometers. Because of the Earth/magnetosphere sweep through the inertial space, the Cluster II satellites every year explore different magnetospheric regions such as the magnetopause, magnetotail, high-altitude auroral region, as well as upstream of the Earth's bow shock. 


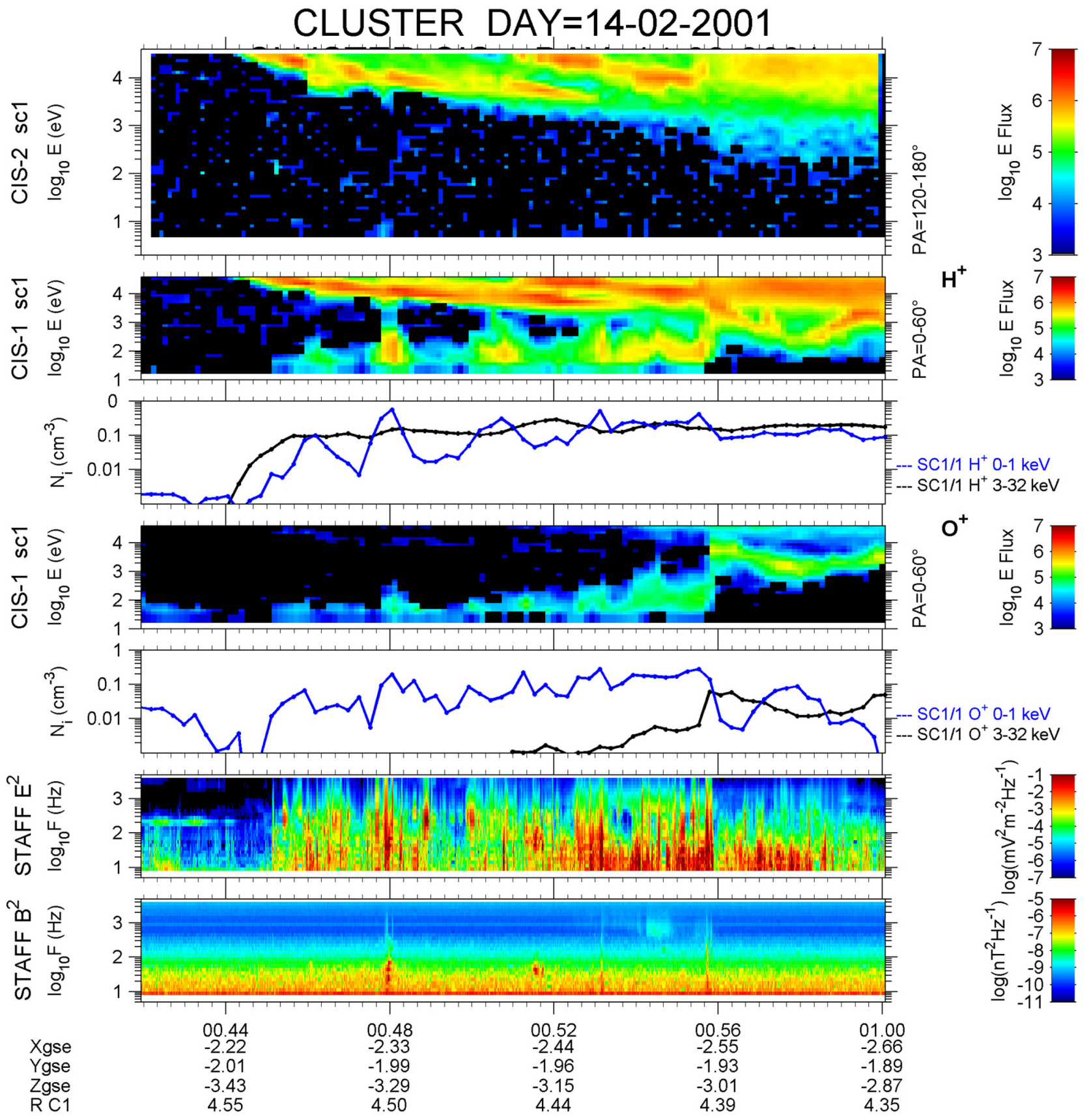

Figure 1. STAFF and CIS measurements from the Cluster 1 satellite are shown from 14 February 2001, southern pass, when the satellite entered the near-Earth plasma sheet boundary layer (PSBL) at about 0045 UT. The first and second panels show the $\mathrm{H}^{+}$ion energy color spectrogram $\left(\log _{10} \mathrm{E}[\mathrm{eV}]\right)$ for pitch angles from $120^{\circ}$ to $180^{\circ}$ (earthward flow) and $0^{\circ}$ to $60^{\circ}$ (tailward flow), respectively. The third panel shows $\mathrm{H}^{+}$density $\left(\mathrm{N}_{\mathrm{i}}\right)$ for low energy $(<1 \mathrm{keV}$, blue curve) and higher energies $(3-32 \mathrm{keV}$, black curve). The fourth and fifth panels show the $\mathrm{O}^{+}$energy spectrogram for tailward flow (pitch angles from $0^{\circ}$ to $60^{\circ}$ ) and the density (blue curve for low energies $<1 \mathrm{keV}$ and black curve for higher energies $3-32 \mathrm{keV}$ ), respectively. The sixth and seventh panels show the electric and magnetic field wave energy $\left(\mathrm{E}^{2}\right.$ and $\left.\mathrm{B}^{2}\right)$ frequency color spectrograms $\left(\log _{10} \mathrm{~F}[\mathrm{~Hz}]\right)$, respectively.

[8] On 14 February 2001 in the southern hemisphere the Cluster II satellites passed from the magnetotail lobe into the near-Earth plasma sheet. This can be seen in Figure 1, which shows, from top to bottom, the CIS instrument [Rème et al., 2001], hydrogen ion energy spectrograms for pitch angles between $120^{\circ}-180^{\circ}$ (earthward flow) and $0^{\circ}-60^{\circ}$ (tailward flow), $\mathrm{H}^{+}$densities, $\mathrm{O}^{+}$energy spectrogram and densities, and electric and magnetic field spectrograms from the STAFF instrument [Cornilleau-Wehrlin et al., 2003], respectively. It can be seen in Figure 1 that after about 0045 UT, the satellite crosses from the higher-latitude lobepolar cap region into the PSBL, as evidenced by the enhanced 

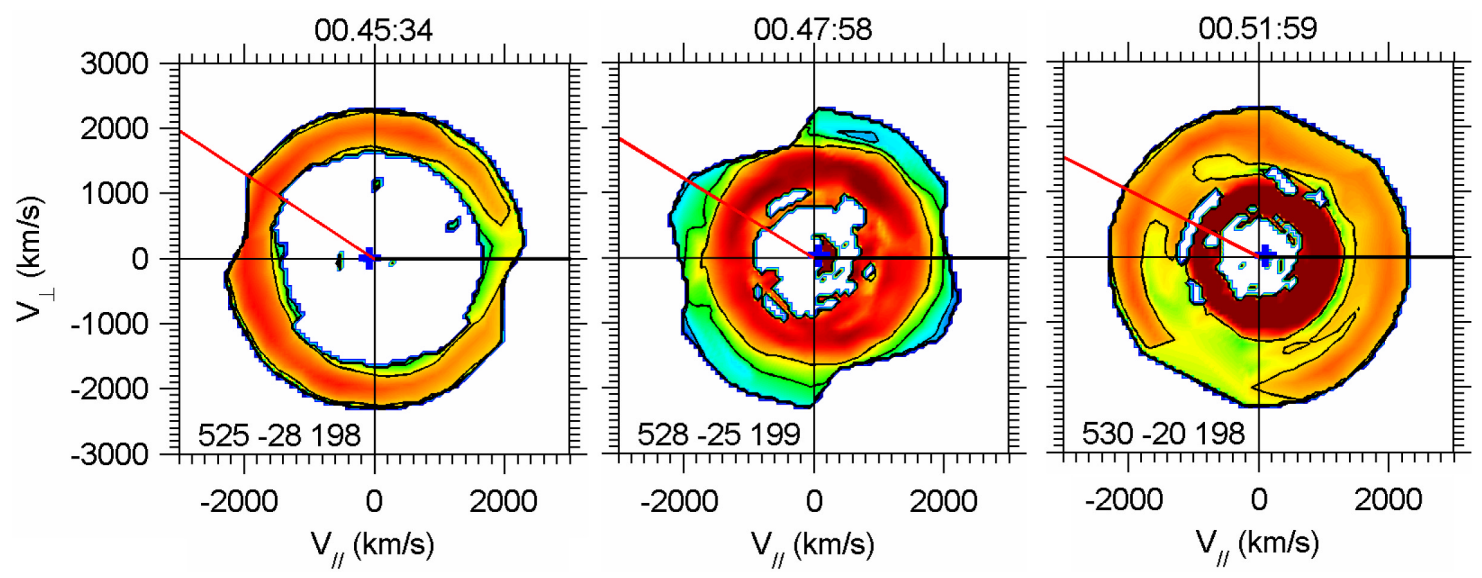

Figure 2. Velocity space contours from the CIS instrument onboard the Cluster 1 satellite at three different times (12 s accumulation) during the interval shown in Figure 1, with (left) 0045:34 UT, (middle) 0047:58 UT, and (right) 0051:59 UT. Perpendicular velocity (in $\mathrm{km} / \mathrm{s}$ ) is plotted versus parallel velocity (in $\mathrm{km} / \mathrm{s}$ ), and the circular patterns seen in the high energy ions are indicative of ion shell distributions. The distribution at 0051:59 UT shows evidence of two shells at different energies.

electric field wave activity (sixth panel) and the appearance of high energy, earthward flowing $\mathrm{H}^{+}>10 \mathrm{keV}$ (first panel). The most intense electrostatic emissions have a somewhat broadband frequency range between about $10 \mathrm{~Hz}$ and $\sim 200 \mathrm{~Hz}$. As the satellite moves to lower latitudes the ion energy decreases, exhibiting the velocity dispersed ion structure (VDIS) pattern that has been observed previously in the near-Earth high-latitude PSBL [Bosqued, 1987; Zelenyi et al.,1990; Bosqued et al., 1993]. This pattern in the electric field and ion energy spectrogram is typical of entry into the PSBL from high latitudes as the satellite traverses toward the central plasma sheet. The $\mathrm{H}^{+}$and $\mathrm{O}^{+}$ energy spectrograms for pitch angles $0^{\circ}-60^{\circ}$ (second and fourth panels) show the presence of low-energy $(<1 \mathrm{keV})$ upgoing ions. The density line plots (third and fifth panels) show the density for lower energy $(<1 \mathrm{keV}$, blue curves) and higher energy ( $>3 \mathrm{keV}$, black curves) and it can be seen that the higher energy portion of the ions are composed almost entirely of $\mathrm{H}^{+}$, whereas the lower-energy ions are a mixture of $\mathrm{O}^{+}$and $\mathrm{H}^{+}$. Thus it can be concluded that the high energy $(>3 \mathrm{keV})$ VDIS $\mathrm{H}^{+}$ions originate from the deeper magnetotail and the low energy $(<1 \mathrm{keV}) \mathrm{O}^{+}$and $\mathrm{H}^{+}$ions flow from below the satellite out of the ionosphere.

[9] Ion distribution function contours are shown in Figure 2 for three selected times during the interval shown in Figures 1 with perpendicular velocity plotted versus parallel velocity (both in $\mathrm{km} / \mathrm{s}$ ). In Figure 2 the high-energy ions form a shell distribution, which describes a plasma in which all of the ions have about the same drift speed isotropically distributed in all directions of velocity space on a sphere with a radius equal to the shell drift speed [e.g., Tataronis and Crawford, 1970a, 1970b]. For example, the distribution taken at 0045:34 UT (Figure 2, left) has a shell drift speed of about $2000 \mathrm{~km} / \mathrm{s}\left(\sim 14 \mathrm{keV}\right.$ for $\left.\mathrm{H}^{+}\right)$. The nature of the shell distribution can be seen more clearly in Figure 3, which shows the ion velocity space distribution at 0047:58 UT for the two transverse velocities plotted versus parallel velocity, duplicated in the first two columns, and the two transverse velocities plotted against each other for three different parallel velocity cuts $(-500,0,+500 \mathrm{~km} / \mathrm{s})$ in the third column. Regardless of which cut is taken in Figure 3, there is a drift velocity in both the perpendicular and parallel directions, clearly showing the shell distribution.

[10] It can be seen in both Figures 2 and 3 for the ion distribution taken at 0047:58 UT that in addition to a highenergy shell, a lower-energy ionospheric ion population is present with a parallel drift speed of about $200 \mathrm{~km} / \mathrm{s}$ directed tailward, out of the ionosphere. There is a correspondence of enhanced electrostatic wave activity seen at this time (Figure 1) with the simultaneous presence of the shell distribution functions and low-energy ions. In general, the strongest wave emissions occur when cold ionospheric plasma is present along with the shells. The ion cyclotron frequency $\left(f_{\text {ci }}\right)$ is $\sim 8 \mathrm{~Hz}$ at the time of observation, which is close to the frequency of the lowest portion of the enhanced wave activity, and the emissions go up to $\sim 200 \mathrm{~Hz}$, which is about $25 f_{\text {ci }}$. A detailed analysis of the wave power during this interval shows that the ratio of the transverse wave intensity to parallel wave intensity $\left(E_{\mathrm{y}} / E_{\mathrm{x}}\right)$ with respect to the ambient magnetic field $\mathbf{B}$ is $>1$. For example at 0048:00 UT, the main peaks in power are at $45 \mathrm{~Hz}, 70 \mathrm{~Hz}$, and $170 \mathrm{~Hz}$ and these peaks have electric field ratios $E_{\mathrm{y}} / E_{\mathrm{x}}=$ $3.7,1.2$ and 1.6, respectively, which shows that the $k$ vector is primarily perpendicular to $\mathbf{B}$. Thus it is concluded that the low-frequency electrostatic emissions observed coincident with the shell distributions are ion Bernstein mode waves excited at harmonics of the ion gyrofrequency.

[11] A close look at the ions and electrons during the Cluster crossing of the southern PSBL on 14 February 2001 shows that the plasma is anisotropic. This can be seen clearly in Figure 4, which shows the electron temperature $(100-500 \mathrm{eV})$ from the 3-D distribution and omnidirectional energy spectrogram of the high-energy sensor (HEEA) from the PEACE instrument [Johnstone et al., 1997] in the first and second panels and the temperature of higher-energy $(1-28 \mathrm{keV})$ and lower-energy $(<1 \mathrm{keV})$ ions in the third and fourth panels, and the energy spectrogram in the fifth panel, respectively. The temperature profiles in the first, third and fourth panels show the parallel temperature $\left(T_{\|}\right)$ and perpendicular temperatures $\left(T_{\perp}\right)$ by the black and blue 


\section{CLUSTER-SC1 CIS2 DAY=14-02-2001 0047:59 UT}
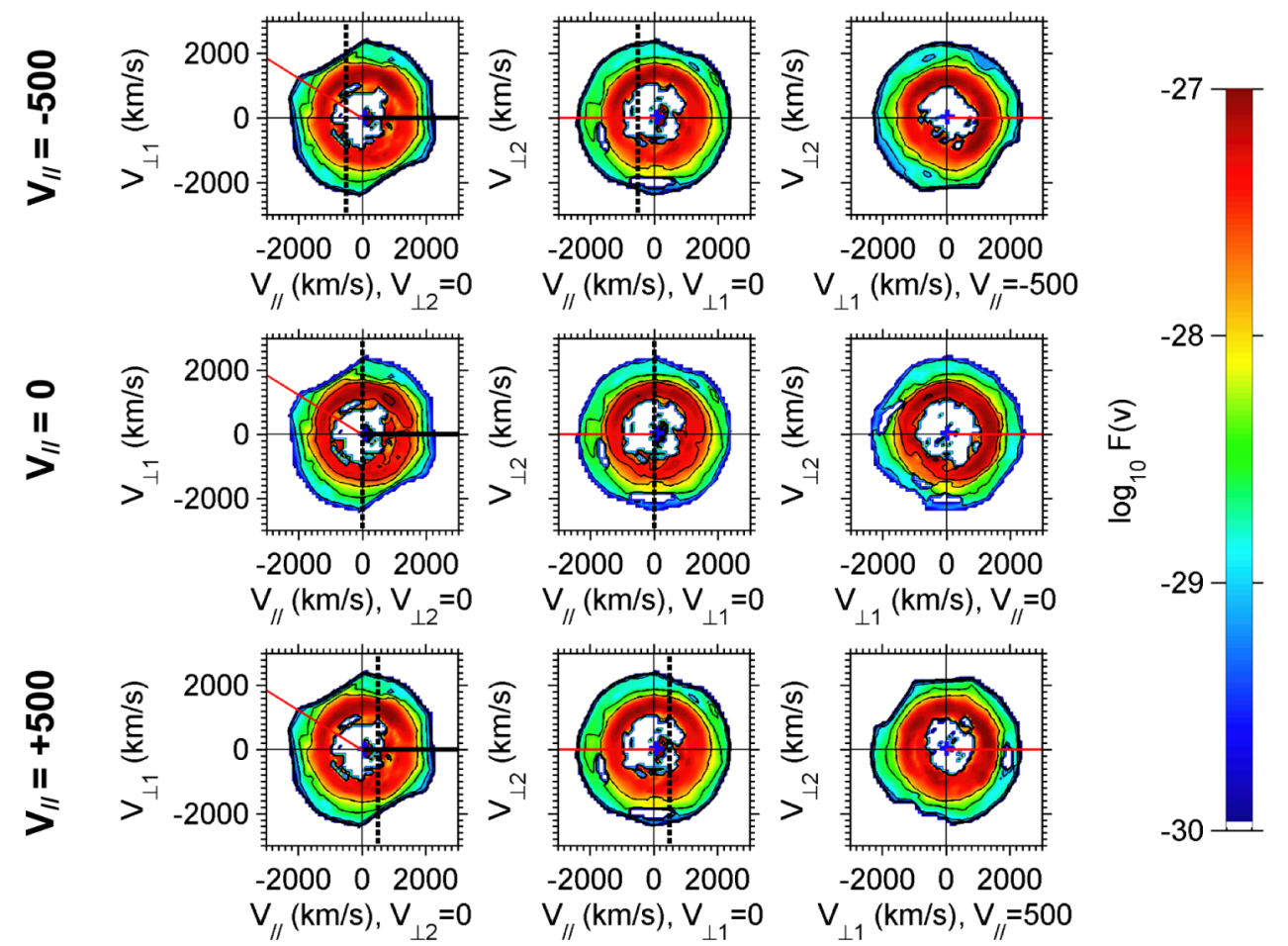

Figure 3. Velocity space contours from the CIS instrument onboard Cluster 1 are presented for the ions at 0047:58 UT (14 February 2001), whereby the first column shows the first transverse velocity plotted versus parallel velocity (duplicated three times), the second column shows the second transverse velocity plotted versus parallel velocity (duplicated three times), and the third column shows the two transverse velocities plotted versus each other for three cuts at three different parallel velocities (top, cut at $\mathrm{V}_{\|}=$ $-500 \mathrm{~km} / \mathrm{s}$; middle, cut at $\mathrm{V}_{\|}=0 \mathrm{~km} / \mathrm{s}$; bottom, cut at $\mathrm{V}_{\|}=+500 \mathrm{~km} / \mathrm{s}$ ).

curves, respectively. It can be seen that for the most part that the electrons have $T_{\|}>T_{\perp}$ (first panel), the high-energy ions are isotropic in temperature with $T_{\perp} \approx T_{\|}$(third panel), and the lower-energy ions at times have $T_{\perp}>T_{\|}$(fourth panel). For example at 00:47:58 UT, coincident with the ion shell distribution, the electrons have a parallel anisotropy with $T_{\|} \sim$ $1.5 T_{\perp}$, while the cold background ions have a perpendicular anisotropy with $T_{\perp} \sim 1.5 T_{\|}$. The observational data presented are consistent with an ion shell instability being excited in the near-Earth PSBL, which will now be explored in detail using linear theory and particle in cell simulations.

\section{Linear Theory}

[12] The analytic linear dispersion relation for the combination of shell ions, cold Maxwellian background ions, and Maxwellian electrons has been obtained, following Tataronis and Crawford [1970a, 1970b] and Sentman et al. [1986, 1987], assuming a cold or delta function ion shell given by:

$$
f_{s}=\left(4 \pi v_{s}^{2}\right)^{-1} \delta\left(v-v_{s}\right)
$$

The delta function shell is a reasonable approximation since in the simulations the directed shell velocity is much larger than the thermal spread of the shell (i.e., $v_{s} \gg v_{\mathrm{ts}}$ ) and for analytic tractability only purely perpendicular propagation with respect to the ambient magnetic field is considered. The linear dispersion relation can then be written as:

$$
\begin{aligned}
1 & -\frac{\omega_{p e}^{2}}{\omega_{c e}^{2}} \sum_{m=-\infty}^{+\infty} \frac{e^{-\left(k_{\perp} v_{t e} / \omega_{c e}\right)^{2}} I_{m}\left[\left(k_{\perp} v_{t e} / \omega_{c e}\right)^{2}\right] \frac{m \omega_{c e}}{\omega-m \omega_{c e}}}{\left(k_{\perp} v_{t e} / \omega_{c e}\right)^{2}} \\
& -\frac{\omega_{p c}^{2}}{\omega_{c i}^{2}} \sum_{m=-\infty}^{+\infty} \frac{e^{-\left(k_{\perp} v_{t c} / \omega_{c i}\right)^{2}} I_{m}\left[\left(k_{\perp} v_{t c} / \omega_{c i}\right)^{2}\right] \frac{m \omega_{c i}}{\omega-m \omega_{c i}}}{\left(k_{\perp} v_{t c} / \omega_{c i}\right)^{2}} \\
& -\frac{\omega_{p s}^{2}}{\omega_{c i}^{2}} \sum_{m=-\infty}^{+\infty} \frac{J_{2 m}\left(2 k_{\perp} v_{s} / \omega_{c i}\right) \frac{m \omega_{c i}}{\omega-m \omega_{c i}}}{\left(k_{\perp} v_{s} / \omega_{c i}\right)^{2}}=0
\end{aligned}
$$

with complex frequency $\omega$, Bessel function $J$ and modified Bessel function $I$ and where the sum is over ion cyclotron harmonic number $m$ and $\omega_{\mathrm{pe}}, \omega_{\mathrm{ps}}$ and $\omega_{\mathrm{pc}}$ are the plasma frequencies of the electrons, shell ions and the cold ions respectively, and $\omega_{\mathrm{ce}}$ and $\omega_{\mathrm{ci}}$ are the electron and ion gyrofrequencies.

[13] The parameters used to find the dispersion relation are deduced from the observations discussed in the previous section. The strength of the magnetic field is such that the electron cyclotron frequency is equal to the electron plasma frequency $\omega_{\text {ce }}=\omega_{\text {pe }}$. The density ratio between shell ions and electrons is $n_{\mathrm{S}} / n_{\mathrm{e}}=2 / 3$ and that between cold background ions and electrons is $n_{\mathrm{c}} / n_{\mathrm{e}}=1 / 3$, so that the density ratio between shell ions and cold ions is $n_{\mathrm{s}} / n_{\mathrm{c}}=2$. The ratio 


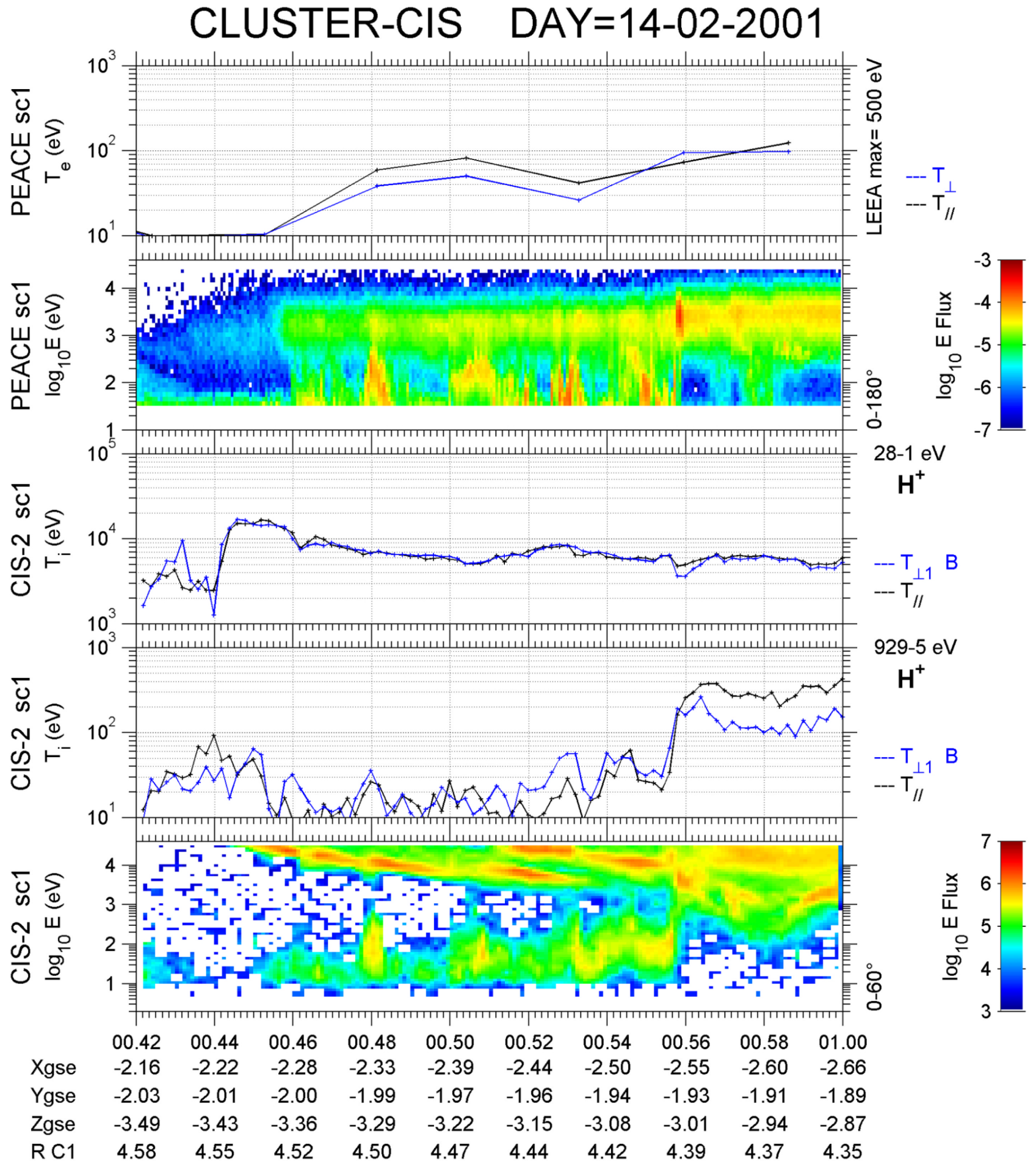

Figure 4. PEACE and CIS data from the 14 February 2001 southern PSBL crossing. The first and second panels show the electron temperature for low energies $(<500 \mathrm{eV})$ and omnidirectional energy spectrogram, respectively, with the black curve in the first panel showing parallel temperature $\left(T_{\|}\right)$and the blue curve showing perpendicular temperature $\left(T_{\perp}\right)$. The third and fourth panels show the $\mathrm{H}^{+}$ temperature for high energies $(1-28 \mathrm{keV})$ and lower energies $(5-929 \mathrm{eV})$, respectively, with the black curve displaying parallel temperature and the blue curve showing perpendicular energy. The sixth panel shows the ion energy spectrogram for pitch angles $0^{\circ}-60^{\circ}$ (tailward flow). 

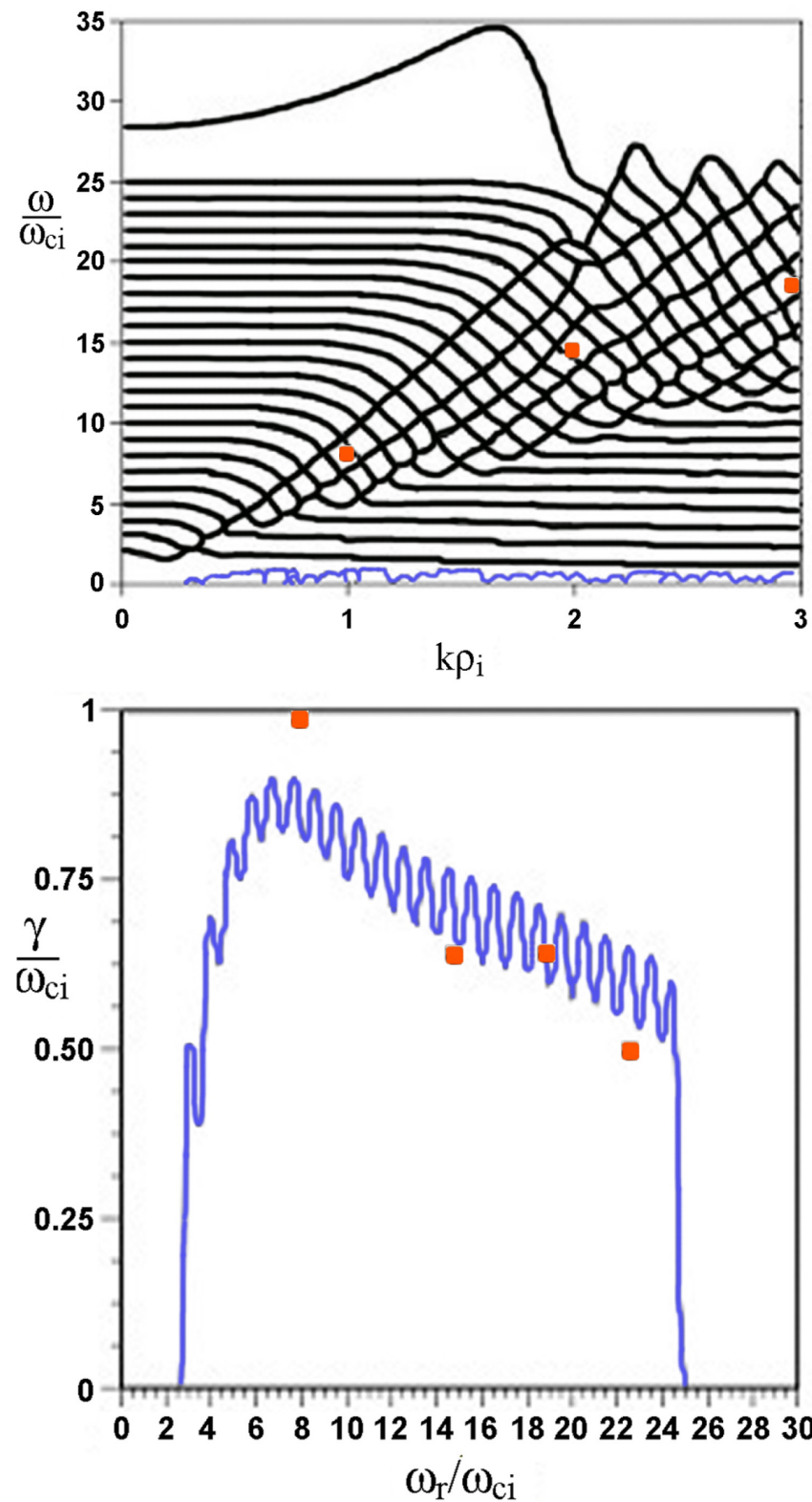

Figure 5. (top) Linear dispersion relation with frequency (normalized to the ion gyrofrequency, $\omega_{\mathrm{ci}}$ ) plotted versus wave number (multiplied times the background ion gyroradius, $\rho_{\mathrm{i}}$ ). Black solid lines show real frequency, and the blue line shows the growth rate. (bottom) Maximum growth rate $\gamma_{\max }$ plotted (in blue) versus real frequency $\omega_{\mathrm{r}}$, both normalized to $\omega_{\text {ci }}$. The red boxes show the results from the simulations, which are discussed in section 5 .

of the ion shell drift velocity to thermal velocity of the cold background ions is $v_{\mathrm{s}} / v_{\mathrm{tc}}=14.1$, with equal shell ion and cold background ion temperatures such that $T_{\mathrm{s}} / T_{\mathrm{c}}=1$ and electron to ion thermal temperature ratios of $T_{\mathrm{e}} / T_{\mathrm{s}}=$ $T_{\mathrm{e}} / T_{\mathrm{c}}=10$. The linear dispersion relation in equation (2) has been solved numerically using these parameters and the resulting roots are displayed in Figure 5, where Figure 5 (top) shows frequency $\left(\omega / \omega_{\text {ci }}\right)$ plotted versus wave number $\left(k_{\perp} \rho_{\mathrm{i}}\right.$, with $\rho_{\mathrm{i}}$ the ion gyroradius of the cold ions) and Figure 5 (bottom) shows growth rate $\left(\gamma / \omega_{\text {ci }}\right)$ versus real frequency $\left(\omega_{\mathrm{r}} / \omega_{\text {ci }}\right)$. In Figure 5 (top) the solid black curves show the real frequency and the blue curve shows the growth rate, respectively. The red boxes on Figure 5 show results from the simulations, which will be discussed in section 5. The richness and complexity of the solutions is apparent on Figure 5 (top), where regions of instability straddle the various ion cyclotron harmonics up to near the plasma frequency of the cold ions $\omega_{\mathrm{pc}} \approx 25 \omega_{\mathrm{ci}}$ along lines corresponding to $\omega_{\mathrm{r}}=k_{\perp} v_{\mathrm{s}}$. Figure 5 clearly shows excitation of waves from $\sim 2 \omega_{\text {ci }}$ up to $\sim 25 \omega_{\text {ci }}$, with robust growth rates $\gamma$ ranging from $0.5-0.9 \omega_{\mathrm{ci}}$ over almost the entire range of excited waves. The upper cutoff of the instability at $25 \omega_{\text {ci }}$, occurs near the cold ion lower hybrid frequency, which is about $27 \omega_{\text {ci }}$, and can be seen in Figure 5 (top) as the dispersion branch at the highest frequency.

[14] We have examined the situation when the cold background ions are removed (i.e., $n_{\mathrm{c}}=0$ ) such that only the ion shell is present along with background electrons, and it was found that there are no unstable wave modes for all other parameters being the same as above. This is consistent with the results of Tataronis and Crawford [1970a, 1970b] that show a shell by itself is inherently stable, but if a cold background is added the shell can become strongly unstable [Sentman et al., 1986]. Thus a key element in driving the shell modes unstable is the presence of the cold background ions. The next step is to run simulations to examine how the shell instability saturates and how the plasma is affected by the ensuing wave-particle interactions.

\section{Simulation Model}

[15] A particle-in-cell (PIC) model with electrostatic fields and with full dynamics ions and drift kinetic electrons has been used to simulate ion ring and shell instabilities in the presence of a background of cold ions and electrons, all embedded in a strong ambient magnetic field [Lee and Okuda, 1978]. The drift kinetic treatment of the electrons entails that the electrons are only accelerated by the electric field along the ambient magnetic field while their perpendicular dynamics is described to lowest order by the $\mathbf{E} \times \mathbf{B}$ drift. This translates into the following equations of motion for ions (subscript i) with position $\mathbf{x}_{\mathbf{i}}$, velocity $\mathbf{v}_{\mathrm{i}}$, and mass $m_{\mathrm{i}}$ :

$$
\begin{gathered}
\frac{d}{d t} \mathbf{x}_{\mathrm{i}}=\mathbf{v}_{\mathrm{i}} \\
\frac{d}{d t} \mathbf{v}_{\mathrm{i}}=\frac{|e|}{m_{\mathrm{i}}}\left(\mathbf{E}+\frac{\mathbf{v}_{\mathrm{i}}}{c} \times \mathbf{B}_{0}\right)
\end{gathered}
$$

For electrons (subscripted by e) with mass $m_{\mathrm{e}}$, the equations of motion are

$$
\begin{aligned}
\frac{d}{d t} \mathrm{v}_{\| \mathrm{e}}=\frac{\mathrm{F}_{\|}}{m_{\mathrm{e}}}=-\frac{|\mathrm{e}|}{m_{\mathrm{e}}} \mathrm{E}_{\|} & =-\frac{|\mathrm{e}|}{m_{\mathrm{e}}}\left(\mathbf{E} \cdot \frac{\mathbf{B}_{0}}{\mathrm{~B}_{0}}\right)=-\frac{|\mathrm{e}|}{m_{\mathrm{e}}}(\mathbf{E} \cdot \hat{\mathbf{b}}) \\
\mathbf{v}_{\perp \mathrm{e}} & =\frac{c}{\mathrm{~B}_{0}^{2}}(\mathbf{E} \times \mathbf{B}) \\
\frac{d}{d t} \mathbf{x}_{\mathrm{e}} & =\mathrm{v}_{\| \mathrm{e}} \hat{\mathbf{b}}+\mathbf{v}_{\perp \mathrm{e}}
\end{aligned}
$$


Here $\|$ and $\perp$ indicate directions parallel and perpendicular to the ambient magnetic field $\mathbf{B}_{0}$, $\hat{\mathbf{b}}$ is the unit vector along that field, $e$ the signed electron charge, $c$ the speed of light, $\mathbf{E}$ the electrostatic field, while $F$ denotes the force, and $t$ indicates time.

[16] In this guiding center model, the particles are of finite size and have been assigned a Gaussian shape on a Cartesian spatial grid where the fields and forces are also distributed and evaluated [Dawson, 1983]. The guiding center model has been used successfully to study drift wave instabilities in strongly magnetized fusion plasmas [Leboeuf et al., 1986]. The drift kinetic description of the electrons means that time steps large compared to the electron cyclotron frequency can be taken so that phenomena on the much longer ion timescales are accessible. However, the drift kinetic nature of the electrons complicates the numerics compared to conventional PIC codes where the full dynamics for both electrons and ions is followed. For these codes, the leapfrog scheme is utilized throughout to integrate the particle orbits in time. The straightforward leapfrog scheme which is time centered and second order accurate in time can still be used in the guiding center codes for the full dynamics ions to advance their velocities and positions in time. However, a combination of leapfrog for parallel acceleration and predictor-corrector for perpendicular motion which preserves time centering has been found most effective to follow the electrons in space and time [Lee and Okuda, 1978; Sydora et al., 1986].

[17] Leapfrog integration of the ion orbits proceeds as follows with positions, charge density and electric field at half time $(n+1 / 2) \Delta t$ and velocities at full time $(n+1) \Delta t$ with time step $\Delta \mathrm{t}$ :

$$
\begin{gathered}
\mathbf{v}_{\mathrm{i}}^{\mathrm{n}+1}=\mathbf{v}_{\mathrm{i}}^{\mathrm{n}}+\Delta t \frac{|e|}{M_{\mathrm{i}}}\left[\mathbf{E}+\frac{\left(\frac{\mathbf{v}_{\mathrm{i}}^{\mathrm{n}+1}+\mathbf{v}_{\mathrm{i}}^{\mathrm{n}}}{2}\right)}{c} \times \mathbf{B}_{0}\right] \\
\mathbf{x}_{\mathrm{i}}^{\mathrm{n}+3 / 2}=\mathbf{x}_{\mathrm{i}}^{\mathrm{n}+1 / 2}+\Delta t \mathbf{v}_{\mathrm{i}}^{\mathrm{n}+1}
\end{gathered}
$$

The velocity at the advanced full time level $n+1$ is solved for implicitly in equation (8) in adherence to the standard Boris pusher [Boris, 1970]. This leads to a time centered, second-order-accurate solution, with the ion density $n_{\mathrm{i}}$ accumulated at the same half time level as the ion positions.

[18] Predictor-corrector integration of the electron trajectories is necessary because their perpendicular velocity is the $\mathbf{E} \times \mathbf{B}$ drift and is therefore known at the same time level as the electron density $n_{\mathrm{e}}$, the electrostatic potential $\phi$ and the electric field. The leapfrog scheme can still be used to advance the parallel part of the equations of motion but midpoint trapezoidal integration is necessary for the perpendicular part in both the predictor (p) step

$$
\begin{gathered}
\mathrm{v}_{\| \mathrm{e}}^{\mathrm{n}+1}=\mathrm{v}_{\| \mathrm{e}}^{\mathrm{n}}+\Delta t \mathrm{~F}_{\| \mathrm{e}}^{\mathrm{n}+1 / 2} \\
\mathbf{x}_{\mathrm{pe}}^{\mathrm{n}+3 / 2}=\mathbf{x}_{\mathrm{e}}^{\mathrm{n}-1 / 2}+2 \Delta t\left[\mathbf{v}_{\perp \mathrm{e}}^{\mathrm{n}+1 / 2}+\hat{\mathbf{b}}\left(\frac{\mathrm{v}_{\perp \mathrm{e}}^{\mathrm{n}+3 / 2}+\mathrm{v}_{\perp \mathrm{e}}^{\mathrm{n}+1 / 2}}{2}\right)\right]
\end{gathered}
$$$$
\mathbf{x}_{\mathrm{ce}}^{\mathrm{n}+3 / 2}=\mathbf{x}_{\mathrm{e}}^{\mathrm{n}+1 / 2}+\Delta t\left[\mathrm{v}_{\| \mathrm{e}}^{\mathrm{n}+1} \hat{\mathbf{b}}+\left(\frac{\mathbf{v}_{\perp \mathrm{e}}^{\mathrm{n}+3 / 2}+\mathbf{v}_{\perp \mathrm{e}}^{\mathrm{n}+1 / 2}}{2}\right)\right]
$$

Like the leapfrog integration of the ions, this predictorcorrector procedure for the electrons rigorously preserves time centering through its elegant and symmetric handling of the parallel velocities in the predictor step and the perpendicular velocities in the corrector step. The penalties are that positions at three half time levels are kept and that the electron density, the electrostatic potential, and the electric field need to be calculated twice per time step, once at the predictor level and another time at the corrector level. Both electron and ion densities are accumulated on the spatial Cartesian grid using the subtracted dipole scheme which is second order accurate in space and leads to minimal numerical dispersion [Kruer et al., 1973; Dawson, 1983]. The forces acting on the particles, which are also calculated on the spatial grid, are applied to the particles using the same subtracted dipole assignment scheme [Dawson, 1983]. The electrostatic potential is obtained from the total charge density $\rho$ through Poisson's equation:

$$
-\nabla \sigma=4 \pi \rho=4 \pi|\mathrm{e}|\left(n_{\mathrm{i}}-n_{\mathrm{e}}\right)
$$

The electric field is given by:

$$
\mathbf{E}=-\nabla \sigma
$$

Poisson's equation is solved in Fourier space for the electrostatic potential $\phi$ using fast Fourier transforms (FFT). Each component of the electric field is then calculated from the directional derivative of the electrostatic potential evaluated using centered finite differences in configuration space. This particular sequence has been shown to be necessary in order to maintain stability in combination with the predictor-corrector electron orbit integration procedure [Byers et al., 1994]. So doing, the overall scheme exhibits superb energy conservation. In fact, total energy is conserved to better than $0.06 \%$ over 162,000 time steps in calculations with realistic ion-toelectron mass ratio of 1600 and just 4 particles per cell.

[19] The computations are performed in a two-dimensional, periodic, spatial box in Cartesian $x-y$ geometry. Electrons and ions are uniformly distributed in space on the Cartesian grid. Velocity space is three-directional. The uniform ambient magnetic field is aligned with the $y$ direction so that the parallel wave vector $k_{\|}=k_{\mathrm{y}}$ and the perpendicular wave vector $k_{\perp}=k_{\mathrm{x}}$. Parallel electron velocities are randomly assigned according to a Maxwellian distribution with thermal velocity $v_{\text {te }}$ with temperature $T_{\mathrm{e}}$. The velocities of the cold background ions in the directions parallel and perpendicular to the uniform ambient magnetic field are also randomly distributed according to a Maxwellian with thermal velocity $v_{\text {tc }}$ with temperature $T_{\mathrm{c}}$ and density $n_{\mathrm{c}}$. The shell ions are randomly assigned to reconstruct an isotropic shell distribution function in velocity space, with shell speed $v_{s}$ and with a Maxwellian 


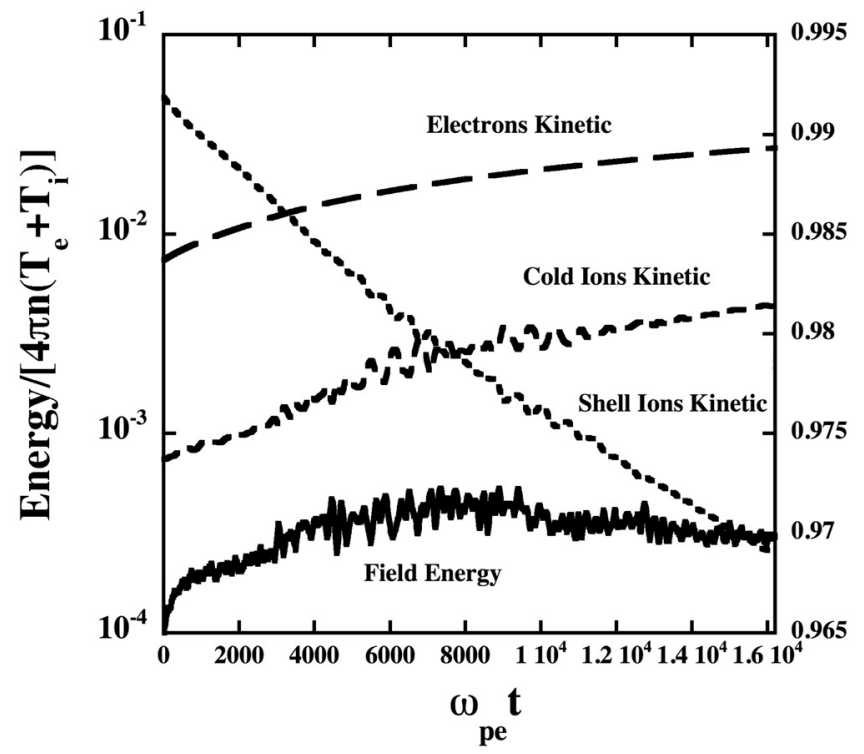

Figure 6. Kinetic energy of the shell ions (dotted curve), kinetic energy of the cold background ions (short-dashed curve), kinetic energy of the electrons (long-dashed curve), and the total electrostatic field energy (solid curve) are plotted versus time during the simulation run. The vertical scale for the electrons, cold ions, and electrostatic field energy is given on the left, while the shell ion kinetic energy scale is given on the right.

thermal spread $v_{\mathrm{ts}}$ at temperature $T_{\mathrm{s}}$ and density $n_{\mathrm{s}}$ of the form [Sentman et al., 1986, 1987]:

$$
f_{\mathrm{s}}=\left(\frac{n_{\mathrm{s}}}{4 \pi^{3 / 2} v_{\mathrm{ts}} v_{\mathrm{s}}^{2}}\right) e^{-\left[\left(v_{\perp}^{2}+v_{\|}^{2}\right)^{1 / 2}-v_{\mathrm{s}}\right]^{2} / 2 v_{\mathrm{ts}}^{2}}
$$

The initial velocity space distribution for the ions includes the ion shell, based on equation (15), and a cold Maxwellian ion background. The spatial grid used in the computations is $\mathrm{N}_{\mathrm{x}} \times \mathrm{N}_{\mathrm{y}}=256 \times 512$, and the number of electrons, equal to the total number of ions, is $\mathrm{N}_{\mathrm{e}}=512 \times 1024$, which translates into 4 electrons and 4 ions per cell. Space is normalized to the electron Debye length $\lambda_{\mathrm{De}}=\mathrm{v}_{\mathrm{te}} / \omega_{\mathrm{pe}}$, so that a unit grid cell is one electron Debye length on each side, velocity is scaled to the electron thermal velocity $v_{\text {te }}$ and time to the plasma frequency $\omega_{\text {pe. }}$. The particle size is taken to be $1.5 \lambda_{\mathrm{De}}$ as is customary in guiding center codes. The time step is such that $\omega_{\mathrm{pe}} \Delta t=0.1$.

\section{Simulation Results}

[20] Using the simulation model described in the previous section with the initial ion distribution function given by equation (15), we have carried out simulation runs of the shell instability. The mass ratio between ions and electrons is $m_{\mathrm{i}} / m_{\mathrm{e}}=1600$ and with this mass ratio, the ion cyclotron frequency is $\omega_{\mathrm{ci}}=6.25 \times 10^{-4} \omega_{\mathrm{pe}}$ and the ratio of the directed ion shell velocity to the electron thermal speed is $v_{\mathrm{s}} / v_{\text {te }}=0.3535$. Moreover the ratio of ion Larmor radius evaluated at the electron temperature to the electron Debye length is $\rho_{\mathrm{i}} / \lambda_{\mathrm{De}}=40$ and that of the Larmor radius of the cold background ions to the electron Debye length is $\rho_{\mathrm{ic}} / \lambda_{\mathrm{De}}=$ 12.65. The computations are carried out for 162,000 time steps, up to time $\omega_{\mathrm{pe}} t=16200$, which corresponds to $\omega_{\mathrm{ci}} t=$ 10.125 and a real time of about $0.2 \mathrm{~s}$.

[21] The time evolution of the kinetic energies of the shell ions, cold background ions, electrons and the electrostatic wave energy is plotted in Figure 6. The scale for the shell ions kinetic energy (dotted curve) is given on the right vertical axis to clearly show the decrease in energy accompanied by the growth of total electrostatic wave energy (solid curve) and the increase in the cold ion background (short-dashed curve) and electron (long-dashed curve) energies.

[22] Figure 7 (top) shows the time history of the total electrostatic wave energy, and Figure 7 (bottom) shows the
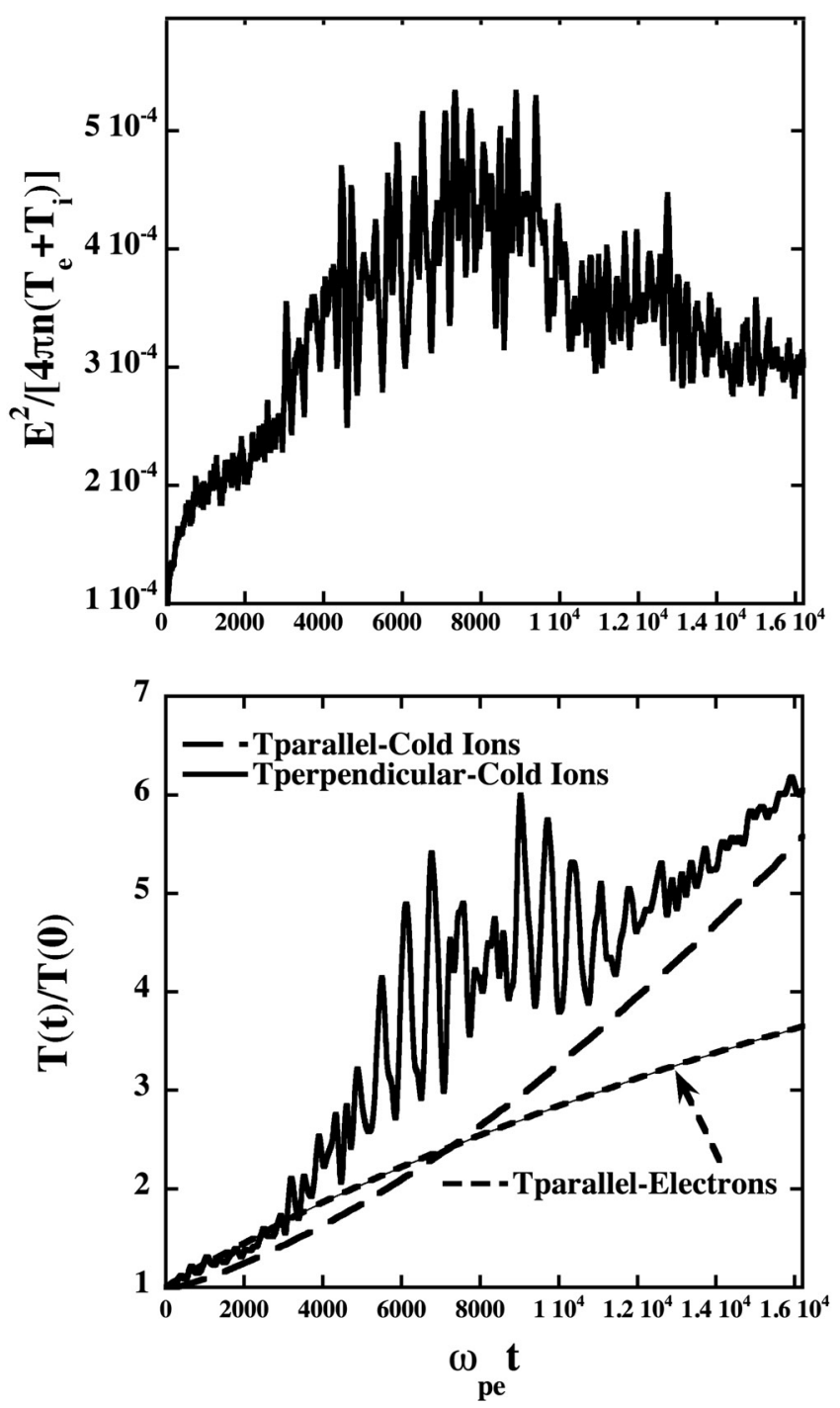

Figure 7. (top) Time history of the electrostatic wave energy summed over all modes and (bottom) time histories of perpendicular (solid curve) and parallel temperatures of the cold ions (long-dashed curve) and the parallel temperature of the electrons (short-dashed curve) normalized to their initial values shown from the simulations. 

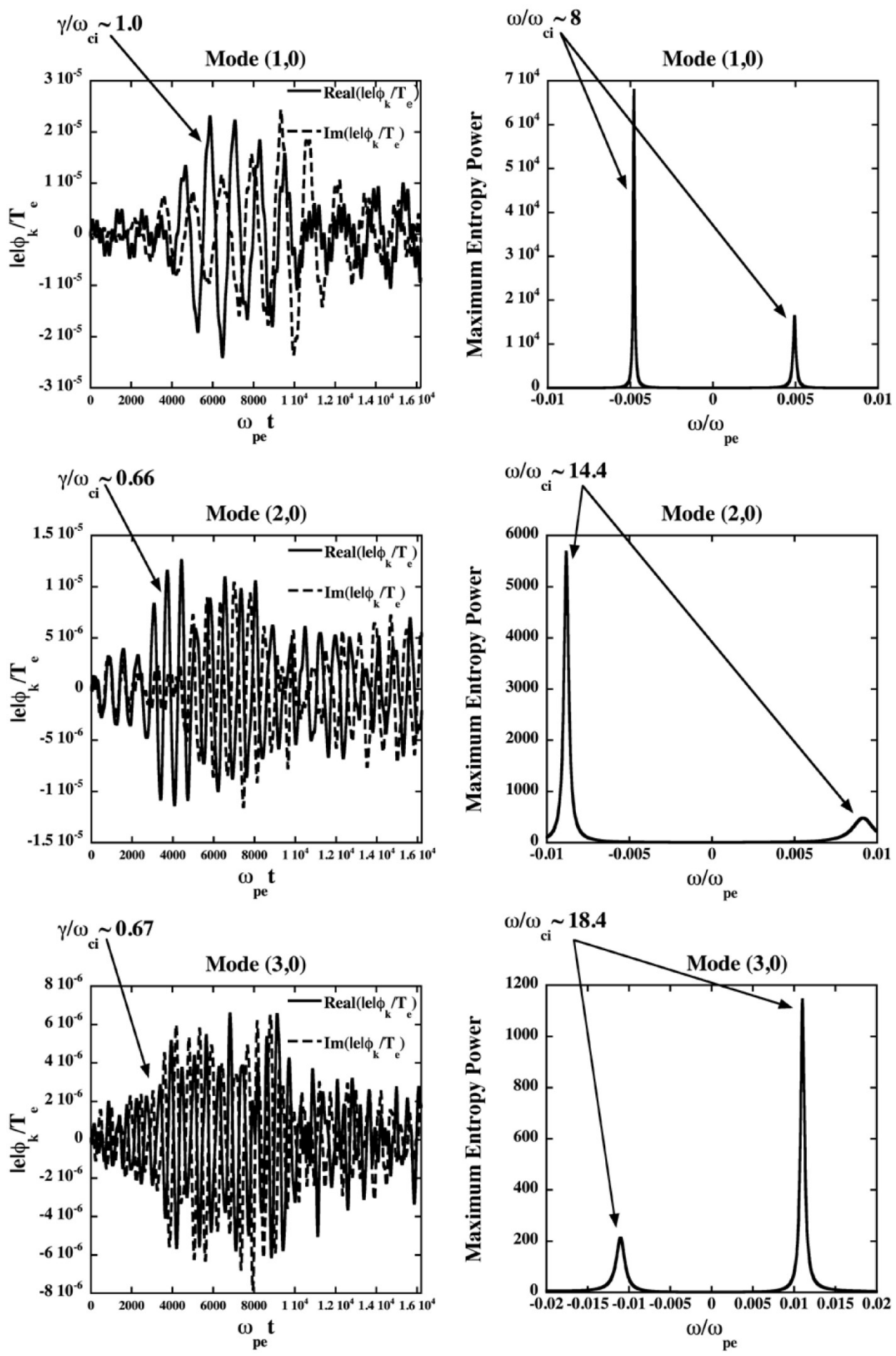

Figure 8. (left) Time history (and measured growth rates) for the first three perpendicular modes from the simulation and (right) the corresponding power spectra (power versus frequency) for each of the three modes.

time histories of the background ion and electron temperatures. The oscillations in time of the perpendicular temperature of the cold ions match well those of the electrostatic energy, indicating a strong coupling between the waves excited by the ion shell instability and the cold background ions. It can be seen that the increase of the perpendicular temperature of the cold ions (solid curve) is proportionally more important than the increases in the parallel temperature of the cold ions (long-dashed curve) and of the electrons (short-dashed curve) with respect to their initial values. 

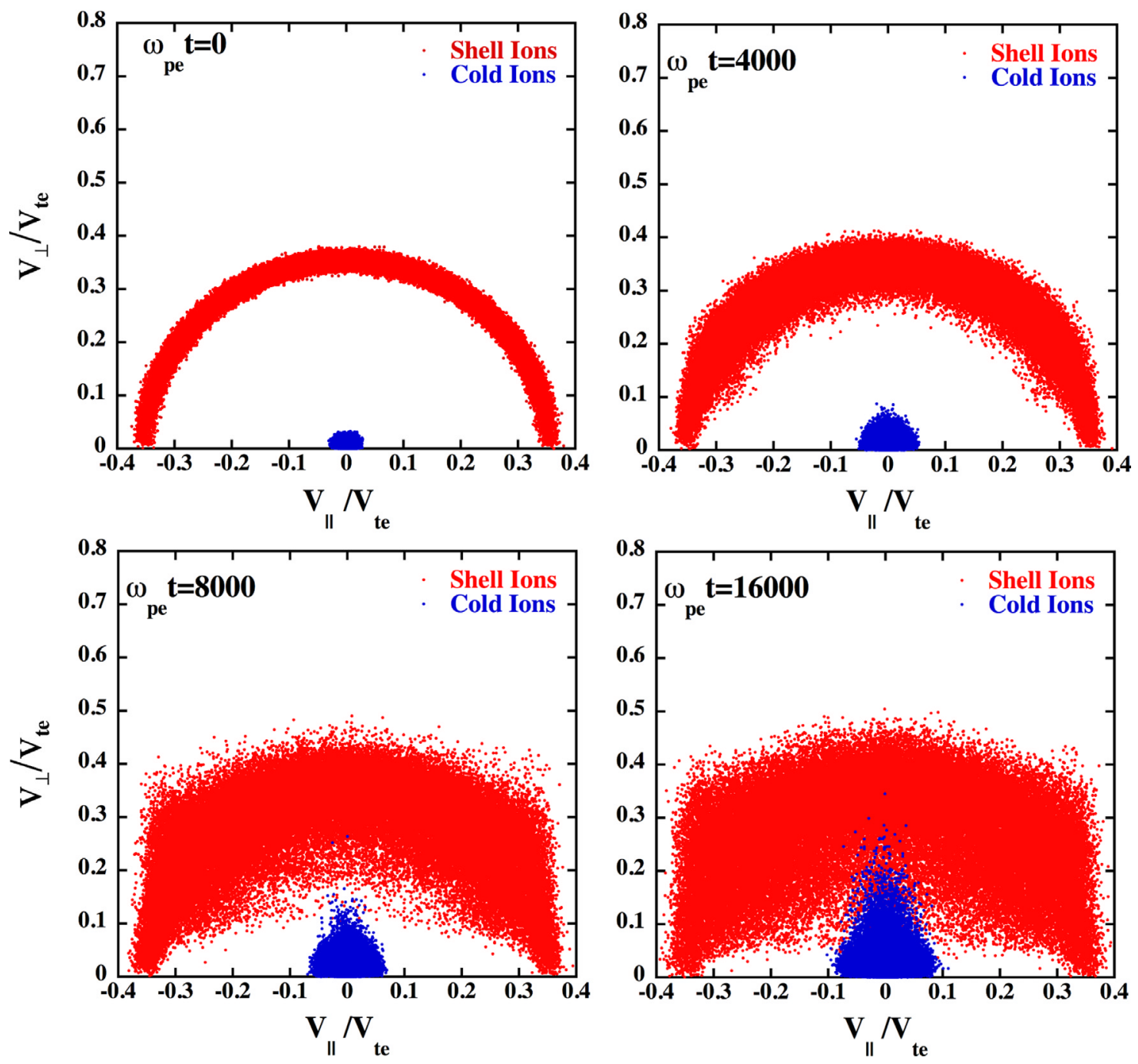

Figure 9. Velocity phase space of ions is shown (perpendicular velocity versus parallel velocity) at four different times during the simulation run for $\omega_{\mathrm{pe}} t=0, \omega_{\mathrm{pe}} t=4000, \omega_{\mathrm{pe}} t=8000$, and $\omega_{\mathrm{pe}} t=16,000$. The shell ions are displayed in red, and the cold background ions are shown in blue.

[23] Detailed time series analysis of the electrostatic potential has been performed. The electrostatic potential is first transformed from configuration space to wave number space using FFTs. This yields the time series at each wave number from which growth rate and real frequency can be extracted (if the signal is monochromatic enough). Then the time series data at each wave number is analyzed by standard signal processing techniques such as FFT [Bendat and Piersol, 2000] and maximum entropy [Burg, 1975] to produce the frequency spectrum and thus provide a direct measurement of the resonant frequency at that particular wave number. These procedures show that the modes with the highest energy and spectral content are those that are purely perpendicular to the magnetic field. This legitimizes our choice of examining these modes when deriving and solving the linear dispersion relation of equation (2).

[24] The time history and power spectrum of the three most energetic wave modes excited in the simulation are shown in Figure 8. The dominant mode is the fundamental wavelength in the direction perpendicular to the magnetic field and corresponds to a wave number of $k_{\perp} \rho_{\mathrm{i}}=0.98$. It is followed in succession by perpendicular mode number 2 and 3 with wave numbers $k_{\perp} \rho_{\mathrm{i}}=1.96$ and $k_{\perp} \rho_{\mathrm{i}}=2.95$. The growth rates are determined from time evolution of the real and imaginary parts of the electrostatic potential and are found to be $\gamma / \omega_{\mathrm{ci}} \approx 1.0$ for the first mode and $\gamma / \omega_{\mathrm{ci}} \approx 0.67$ for modes 2 and 3 . The maximum entropy power spectrum displayed in Figure 8 (right) reveals that the dominant mode (perpendicular wave number mode 1) has a real frequency of $\omega_{\mathrm{r}} \approx 8 \omega_{\mathrm{ci}}$. The higher wave number modes resonate with higher cyclotron harmonics. For instance, the mode numbers 2 and 3 have real frequencies $\omega_{\mathrm{r}} \approx 14.4 \omega_{\mathrm{ci}}$ and $\omega_{\mathrm{r}} \approx 18.4 \omega_{\mathrm{ci}}$, respectively. The growth rates and real frequencies obtained for the energetically relevant modes in the computations are compared to the solutions of the linear dispersion relation shown in Figure 5 (simulation results are indicated by the red boxes in Figure 5) and excellent agreement is found between the two.

[25] As a result of the shell instability being excited there is in an increase of the temperatures of the 


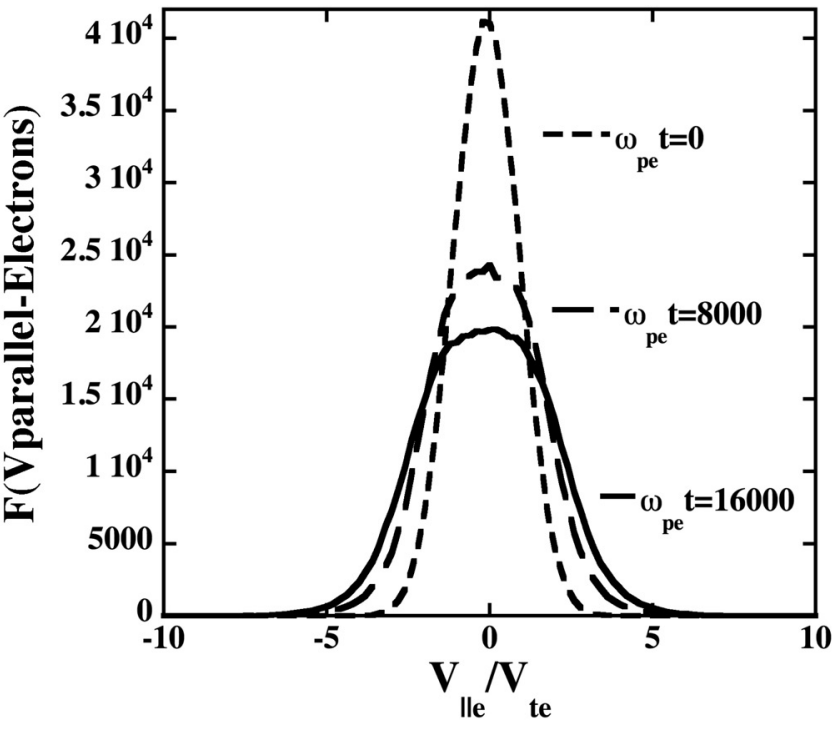

Figure 10. Electron parallel distribution function at three different times during the simulation run, with the shortdashed curve showing the initial state $\left(\omega_{\mathrm{pe}} t=0\right)$, the longdashed curve showing the distribution at $\omega_{\mathrm{pe}} t=8000$, and the solid curve showing $\omega_{\mathrm{pe}} t=16,000$.

background plasma (Figures 6 and 7). A detailed look at the transfer of energy from the waves into the ions can be seen in Figure 9, which shows a plot of the evolution of the velocity space for ions, with snapshots shown at different times from the initial state (Figure 9, top left) to the final state (Figure 9, bottom right). It can be seen that the background ions (blue particles) are heated preferentially in the perpendicular direction, while the shell (red particles) tends to spread in velocity space such that the shell drift energy decreases and is transferred into thermal energy of the shell.

[26] The transfer of energy into electrons is illustrated in Figure 10, which shows the parallel velocity distribution function at three different times during the simulation run. It can be seen in Figure 10 that tail heating happens first as the waves interact with the electrons followed by significant bulk heating as the instability saturates. The electron heating in the simulation can only be in the direction parallel to the magnetic field for the drift kinetic electron approximation and this has to occur through resonant interaction of the electrons with nonlinearly excited waves which propagate obliquely with respect to or along the ambient magnetic field, i.e., waves which have a finite parallel wave vector. Parallel electron heating by ion shell instabilities has also been found in the simulation by Janhunen et al. [2003a].

[27] Although the electron heating is significant, the increase in the perpendicular temperature of the cold background ions is proportionally larger as illustrated in Figure 7. Sentman et al. [1986, 1987] identified the cause of such an energy increase in the background plasma for the case of an electron shell and a cold electron background as a nonstochastic, linear cyclotron heating mechanism by unstable waves at harmonics of the cyclotron frequency. An asymptotic perpendicular velocity $v_{0}$ for the cold particles due to this mechanism was derived and in the case of cold ions can be written as

$$
v_{0} / v_{p h}=\left(\frac{k_{\perp} v_{0}}{\omega_{c i}}\right)\left(\frac{\omega_{c i}}{\omega_{r}}\right) \approx \frac{\pi}{2} \frac{(3 / 2+\mathrm{m})}{\mathrm{m}}
$$

Here $v_{0}$ is given with respect to the perpendicular phase velocity (i.e., $v_{\mathrm{ph}}=\omega_{\mathrm{r}} / k_{\perp}$ ) of the dominant wave mode,. The right hand side of equation (16) corresponds to the first zero of the Bessel function $J_{\mathrm{m}}$ where $m$ is the harmonic of the cyclotron frequency closest to $\omega_{\mathrm{r}} / \omega_{\mathrm{ci}}$. The maximum perpendicular velocity achieved by the cold ions in the simulations is found to be $0.35 v_{\text {te }}$, which can be seen in the perpendicular-parallel velocity phase space displayed in Figure 11 at $\omega_{\text {pe }} t=16000$. The asymptotic perpendicular velocity of the cold ions for the most dominant wave mode with $m=8, \omega_{\mathrm{r}} / \omega_{\mathrm{ci}} \approx 8$, and $k_{\perp} \lambda_{\mathrm{De}}=0.0245$ is calculated to be $v_{0} \sim 0.38 v_{\text {te }}$ from equation (16). Therefore perpendicular heating of the cold ions can be attributed to the linear nonstochastic heating mechanism by ion cyclotron waves. This mechanism was invoked to explain heating of auroral ions by electrostatic ion cyclotron waves [Lysak et al., 1980] and appears to be equally applicable to heating of the cold background ions in the context of ion shell instabilities in the PSBL.

\section{Summary and Conclusions}

[28] We start by first summarizing the Cluster observations. When looking at distribution functions of VDIS in the

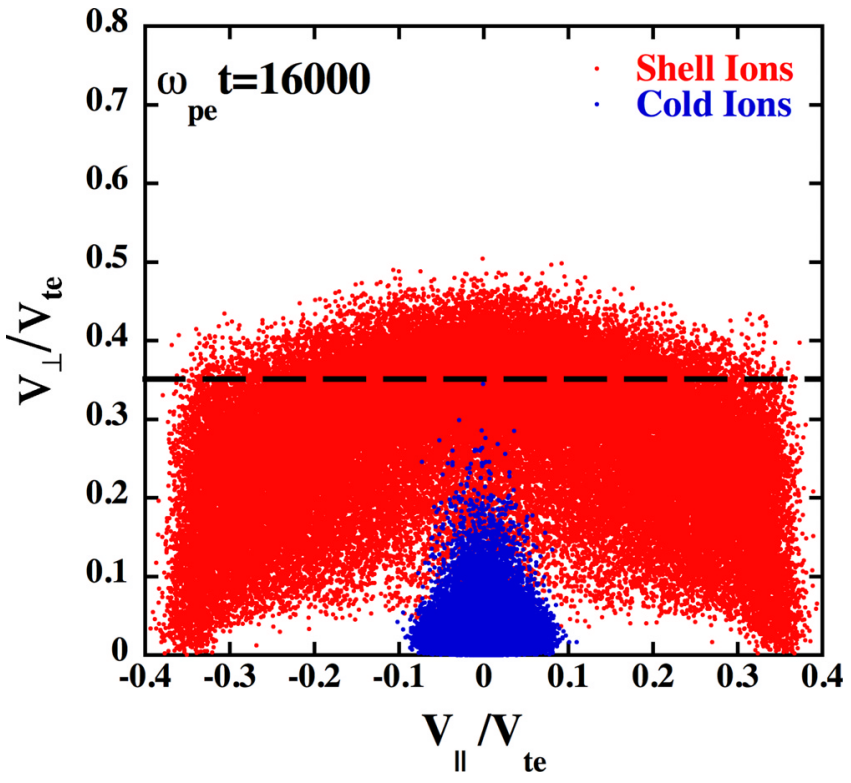

Figure 11. Perpendicular velocity versus parallel velocity phase space at time $\omega_{\text {pe }} t=16,000$ from the simulations, with shell ions in red and background ions in blue. The dashed horizontal line at $0.35 v_{\text {te }}$ shows the highest perpendicular velocity reached by the background ions, which is in good agreement with the expected saturation velocity given by equation (16) of $0.38 v_{\text {te }}$ for the linear nonstochastic ion cyclotron heating mechanism. 


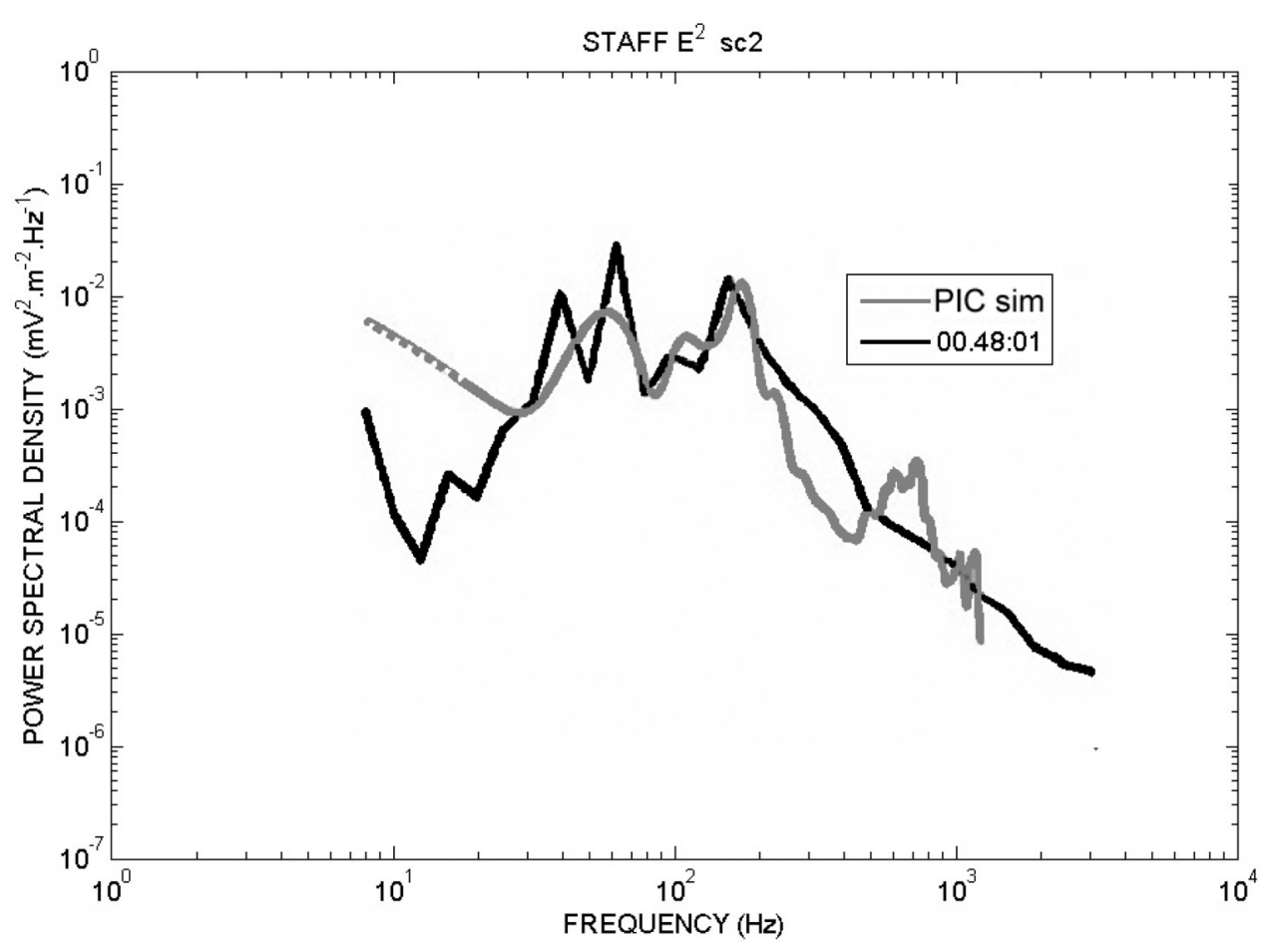

Figure 12. Observed wave power spectral density (black curve) versus frequency from the STAFF instrument during the 14 February 2001 PSBL crossing of the Cluster satellite at 0048:01 UT. The simulated power spectrum is shown by the gray curve. For both curves, peaks appear between about 40 and $70 \mathrm{~Hz}$, and secondary peaks appear at about $100-300 \mathrm{~Hz}$.

Earth's PSBL, observations show that the ions took the form of shell distributions whereby the ions have a drift speed distributed isotropically on a shell in the parallel and perpendicular directions. Ion shell distributions have been observed previously in the near-Earth PSBL by the Polar satellite at somewhat lower altitudes $\sim 18,000 \mathrm{~km}$ [Janhunen et al., 2003a; Olsson et al., 2004] compared to where the Cluster observations were made $(\sim 22,000 \mathrm{~km})$. At certain times during the Cluster PSBL crossing from high to low latitudes, a cold ionospheric plasma background was present along with the shell distributions and at these times a relatively intense, somewhat broadband $(10 \mathrm{~Hz}-$ $300 \mathrm{~Hz}$ ) wave spectrum was also observed. The wave power spectral density versus frequency is shown in Figure 12 from the STAFF instrument taken on 14 February 2001 at 00:48:01 UT indicated by the black curve. The simulated power spectrum curve is shown by the gray curve and it can be seen that the peak in wave power maximizes between about $40 \mathrm{~Hz}$ and $200 \mathrm{~Hz}$ (note that the hydrogen cyclotron frequency is about $8 \mathrm{~Hz}$ at this time). As discussed in section 2, the observed emissions from Cluster have peaks in electric field transverse to the ambient magnetic field (B) such that the $k$ vector is mainly perpendicular to $\mathbf{B}$, indicative of ion Bernstein mode waves. For the observed spectrum in Figure 12, the transverse to parallel electric field values are $E_{\mathrm{y}} / E_{\mathrm{x}}=1.4,1.1$, and 2.3 for the peaks in wave power at $44 \mathrm{~Hz}, 70 \mathrm{~Hz}$ and $176 \mathrm{~Hz}$, respectively. In general, there is excellent correspondence between the observed and simulated power spectrum in terms of where the peaks occur and the relative intensity between the different peaks at harmonics of the cyclotron frequency. The background ion distribution, which is composed of a mixture of hydrogen and oxygen ions, was observed to be anisotropic such that $T_{\perp}>T_{\|}$:

[29] A linear analysis of ion shell distributions using parameters based on the Cluster observations shows that a broadband wave spectrum is unstable to a series of ion cyclotron harmonics with frequencies between about $2 \omega_{\mathrm{ci}}$ and $25 \omega_{\mathrm{ci}}$, which equates to a frequency range between about $10 \mathrm{~Hz}$ to $300 \mathrm{~Hz}$. Numerical simulations of an ion shell distribution show that a broadband wave spectrum of perpendicular, ion cyclotron waves is excited in the same frequency range as found in the linear theory, which results in heating of the cold background ions and background electrons, as well as thermalization of the shell. Since the strongest waves are mainly perpendicular to the ambient magnetic field, the background ions are heated primarily in the transverse direction leading to an anisotropic distribution such that $T_{\perp}>T_{\|}$. Although the perpendicular wave modes are most strongly unstable and dominate the wave spectrum, some power is generated in oblique wave modes that have a finite parallel wave number; this leads to parallel background ion heating and also parallel heating of the electrons. The shell instability saturates primarily by linear nonstochastic ion cyclotron heating of the background ions.

[30] The observations and theoretical findings discussed here tend to support the following scenario in the PSBL. Ions are accelerated in the deep magnetotail, most likely by nonadiabatic processes, which stream earthward as beams in 


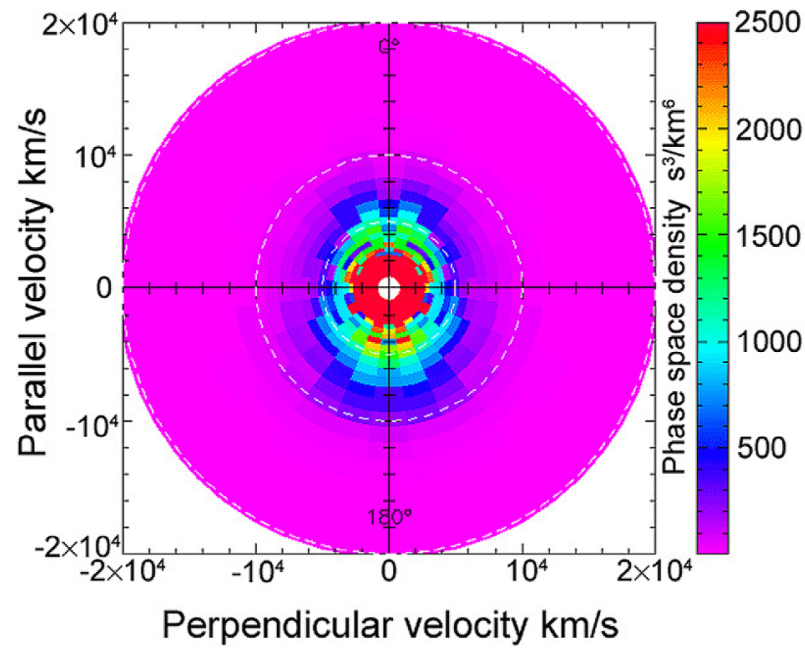

Figure 13. Electron phase space density from the PEACE instrument onboard the Cluster 1 spacecraft shown for the PSBL crossing on 14 February 2001, 0047:58 UT. Parallel velocity in $\mathrm{km} / \mathrm{s}$ on the vertical scale is plotted versus perpendicular velocity in $\mathrm{km} / \mathrm{s}$ on the horizontal scale.

the PSBL. As the beams get close to the Earth, the magnetic mirror effect causes an increase in the pitch angle and takes the beam, which may already have an enhanced perpendicular velocity due to nonadiabatic or wave-particle interactions, and creates a shell distribution in the near-Earth PSBL (i.e., distances from the Earth $\leq 8 \mathrm{R}_{\mathrm{E}}$ ). The region where the ion shell distribution forms is magnetically connected to the auroral region at low altitudes where cold ionospheric ions flow out toward the magnetotail. The outflowing ionospheric ions form a cold background for the ion shell, which then becomes unstable to a broadband, intense spectrum of ion cyclotron waves primarily perpendicular to the magnetic field, but with some nonlinearly excited oblique component as well. These waves extract energy from the ion shell and heat the cold background ions preferentially in the perpendicular direction such that the background ions become anisotropic with $T_{\perp}>T_{\|}$. At higher altitudes the mirror force would tend to turn this increased perpendicular ion energy to parallel ion energy, the net effect of which should be to increase the energy content of the ion beam rather smoothly as a function of altitude. Statistical observational indications for such an increase between 4 and $6 \mathrm{R}_{\mathrm{E}}$ radial distance were reported by Janhunen et al. [2003b].

[31] The shell instability causes the strongly magnetized electrons in the PSBL to be heated primarily in the parallel direction such that they have $T_{\|}>T_{\perp}$. This last conclusion is supported by electron data from the PEACE instrument onboard the Cluster 1 spacecraft shown in Figure 13 for the same PSBL crossing interval considered on 14 February 2001. It can be seen in Figure 13 that the electrons are heated symmetrically in the parallel direction, in qualitative agreement with the simulation results in Figure 11 that show the shell instability results in symmetric bulk heating of the electron distribution.

[32] The excitation of the shell instability in the nearEarth PSBL may have important consequences for large- scale plasma transport in the region. One is the heating of electrons that results in an anisotropic electron distribution with $T_{\|}>T_{\perp}$, which may account for such distributions that are commonly observed in the PSBL. For example electrons in the magnetotail PSBL typically have $T_{\|} / T_{\perp} \sim 1.5$ [Frank et al., 1981; Frank, 1985] and distributions with anisotropies as large as $T_{\|} / T_{\perp} \sim 2$ to 3 have been reported [Hada et al., 1981]. The significance of such an anisotropy is that this can lead to ions and electrons having different mirror points closer to the Earth causing a large-scale charge separation and a parallel potential drop to occur [Alfvén and Fälthammar, 1963; Persson, 1963]. In the case of an electron distribution function having $T_{\|}>T_{\perp}$, the potential drop is negative such that the quasi-static parallel electric field points earthward [Schriver et al., 2001]. If this type of parallel potential drop forms in the primary current region above where a U-shaped potential exists, ultimately an Oshaped potential would form with a negatively charged cloud at the center of the $\mathrm{O}$ [Janhunen and Olsson, 2000; Olsson et al., 2004].

[33] Another consequence of the shell instability is the formation of gaps or breaks in the VDIS. Often referred to as beamlets, it has been suggested that they form due to nonadiabatic resonances in the magnetotail that lead to a mosaic structure of ions in the PSBL [Ashour-Abdalla et al., 1995, 2005]. An alternative to this is that the gaps in the VDIS (and thus the beamlets) are formed by the shell instability. As has been discussed above, the shell instability occurs only when a significant cold background ion population is present and looking at Figure 1, this tends to occur only at particular times during a PSBL crossing. Thus when the cold ion background is present, the shell instability fires causing the shell distribution to lose energy and become more diffuse in velocity as seen in Figure 11. This leads to an overall decrease in the ion energy flux at that location and thus a flux decrease or gap when looking at VDIS in the ion energy spectrogram such as seen in Figure 1. For the event we have examined here on February 14, 2001 there is a direct correspondence between gaps in VDIS and outflowing cold ions. A recent statistical study has shown that there is often a correlation between ion beamlets and electrostatic waves in the frequency range expected from shell instabilities [Janhunen et al., 2005], although it is unclear from this study whether cold background ions, which in most cases are necessary for the shell instability, were present as well.

[34] The study carried out here, as well as previous ion shell instability studies in the PSBL [e.g., Janhunen et al., 2003a] have focused on electrostatic waves. The STAFF Cluster observations (Figure 1), however, show that electromagnetic emissions are also observed coincident with ion shell distributions. It remains to be seen whether ion shells are the cause of these emissions or if shells may be related to kinetic Alfvén waves commonly observed in the PSBL [e.g., Wygant et al., 2000]. We are in the process of examining electromagnetic aspects of ion shell distributions (using the simulation formalism of Geary et al. [1986]) and will report on these findings in the near future.

[35] Acknowledgments. This work was sponsored by NASA grants NAG 5-11923 and NNG05GG58G, NSF grant ATM 02-08507, and DOE grant DE-FG02-ER54740. Work at CESR/CNRS was supported by CNES 
(French Space Agency). We would like to thank David An for his help with the linear dispersion relation. His work was supported by a Summer Internship from the Plasma Science and Technology Institute (PSTI) at UCLA. Computing at UCLA was carried out at the San Diego Supercomputing Center (SDSC) awarded by the National Resource Allocations Committee (NRAC).

[36] Zuyin $\mathrm{Pu}$ thanks Pekka Janhunen and another referee for their assistance in evaluating this paper.

\section{References}

Alfvén, H., and C. G. Fälthammar (1963), Cosmical Electrodynamics, Clarendon, Oxford, U. K

Ashour-Abdalla, M., and H. Okuda (1986), Theory and simulations of broadband electrostatic noise in the geomagnetic tail, J. Geophys. Res. 91, 6833.

Ashour-Abdalla, M., J. Berchem, J. Büchner, and L. M. Zelenyi (1991), Large and small scale structures in the plasma sheet: A signature of chaotic motion and resonance effects, Geophys. Res. Lett., 18, 1603

Ashour-Abdalla, M., J. P. Berchem, J. Büchner, and L. M. Zelenyi (1993), Shaping of the magnetotail from the mantle: Global and local structuring, J. Geophys. Res., 98, 5651.

Ashour-Abdalla, M., L. M. Zelenyi, V. Peroomian, R. L. Richard, and J. M. Bosqued (1995), The mosaic structure of plasma bulk flows in the Earth's magnetotail, J. Geophys. Res., 100, 19,191.

Ashour-Abdalla, M., J. Bosqued, M. El-Alaoui, V. Peroomian, L. M. Zelenyi, R. J. Walker, and J. Wright (2005), A stochastic sea: The source of plasma sheet boundary layer ion structures observed by Cluster, J. Geophys. Res., 110, A12221, doi:10.1029/2005JA011183.

Bendat, J. S., and A. G. Piersol (2000), Random Data: Analysis \& Measurement Procedures, 3rd ed., John Wiley, Hoboken, N. J.

Boris, J. P. (1970), Relativistic plasma simulation - optimization of a hybrid code, in Proceedings of Fourth Conference on Numerical Simulation of Plasmas, p. 3, Nav. Res. Lab., Washington, D. C.

Bosqued, J. M. (1987), AUREOL-3 results on ion precipitation, Phys. Scr. 18,158 .

Bosqued, J. M., M. Ashour-Abdalla, M. El Alaoui, V. Peroomian, L. M. Zelenyi, and C. P. Escoubet (1993), Dispersed ion structures at the poleward edge of the auroral oval: Low-altitude observations and numerical modeling, J. Geophys. Res., 98, 19,181.

Burg, J. P. (1975), Maximum entropy spectral analysis, Ph.D. thesis, Dept. of Geophys., Stanford Univ., Stanford, Calif.

Byers, J. A., A. M. Dimits, Y. Matsuda, and A. B. Langdon (1994), Numerical instability in a 2D gyrokinetic code caused by divergent ExB flow, J. Comput. Phys., 115, 352.

Cattell, C. A., F. S. Mozer, E. W. Hones Jr., R. R. Anderson, and R. D. Sharp (1986), ISEE observations of the plasma sheet boundary layer, plasma sheet, and neutral sheet, 2. Waves, J. Geophys. Res., 91, 5681.

Cornilleau-Wehrlin, N., et al. (2003), First results obtained by the Cluster STAFF experiment, Ann. Geophys., 21, 437-456.

Dawson, J. M. (1983), Particle simulation of plasmas, Rev. Mod. Phys., 55, 403

DeCoster, R. J., and L. A. Frank (1979), Observations pertaining to the dynamics of the plasma sheet, J. Geophys. Res., 84, 5099.

Eastman, T. E., L. A. Frank, W. K. Peterson, and W. Lennartsson (1984), The plasma sheet boundary layer, J. Geophys. Res., 89, 1553.

Forbes, T. G., E. W. Hones Jr., S. J. Bame, J. R. Asbridge, G. Paschmann, N. Sckopke, and C. T. Russell (1981), Evidence for the tailward retreat of a magnetic neutral line in the magnetotail during substorm recovery, Geophys. Res. Lett., 8, 261.

Frank, L. A. (1985), Plasmas in the Earth's magnetotail, Space Sci. Rev., 42 , 211

Frank, L. A., K. L. Ackerson, and R. P. Lepping (1976), On hot tenuous plasmas, fireballs, and boundary layers in the Earth's magnetotail, J. Geophys. Res., 81, 5859.

Frank, L. A., R. L. McPherron, R. J. DeCoster, B. G. Burek, K. L. Ackerson, and C. T. Russell (1981), Field-aligned currents in the Earth's magnetosphere, J. Geophys. Res., 86, 687.

Freund, H. P., and C. S. Wu (1988), Stability of a spherical shell distribution of pickup ions, J. Geophys. Res., 93, 14,277.

Geary, J. L., T. Tajima, J. N. Leboeuf, E. G. Zaidman, and J. H. Han (1986), Two- and three-dimensional magnetoinductive particle codes with guiding center electron motion, Comput. Phys. Comm., 42, 313.

Grabbe, C. L. (2002), Solitary wave structure in magnetized plasmas and the source region of BEN, Geophys. Res. Lett., 29(16), 1804 , doi:10.1029/2002GL015265.

Grabbe, C. L., and T. E. Eastman (1984), Generation of broadband electrostatic noise by ion beam instabilities in the magnetotail, J. Geophys. Res., $89,3865$.

Gurnett, D. A., L. A. Frank, and R. P. Lepping (1976), Plasma waves in the distant magnetotail, J. Geophys. Res., 81, 6059.
Hada, T., A. Nishida, and T. Terasawa (1981), Bi-directional electron pitch angle anisotropy in the plasma sheet, J. Geophys. Res., 86, 11,211.

Hones, E. W., Jr., J. R. Asbridge, S. J. Bame, M. D. Montgomery, S. Singer, and S.-I. Akasofu (1972), Measurements of magnetotail plasma flow made with Vela 4B, J. Geophys. Res., 77, 5503.

Janhunen, P., and A. Olsson (2000), New model for auroral acceleration: O-shaped potential structure cooperation with waves, Ann. Geophys., 18 , 596.

Janhunen, P., A. Olsson, H. Laakso, A. Vaivads, and W. K. Peterson (2003a), Generation of Bernstein waves by ion shell distribution in the auroral region, Ann. Geophys., 21, 881 .

Janhunen, P., A. Olsson, and W. K. Peterson (2003b), The occurrence frequency of upward ion beams in the auroral zone as a function of altitude using Polar/TIMAS and DE-1/EICS data, Ann. Geophys., 21, 2059.

Janhunen, P., A. Olsson, W. K. Peterson, and J. D. Menietti (2005), Latitude-energy structure of multiple ion beamlets in Polar/TIMAS data in plasma sheet boundary layer and boundary plasma sheet below $6 \mathrm{R}_{\mathrm{E}}$ radial distance: Basic properties and statistical analysis, Ann. Geophys., 23,867

Johnstone, A. D., et al. (1997), PEACE: A Plasma Electron and Current Experiment, Space Sci. Rev., 79, 351

Kojima, H., Y. Omura, H. Matsumoto, K. Miyaguti, and T. Mukai (1999), Characteristics of electrostatic solitary waves observed in the plasma sheet boundary: Statistical analyses, Nonlinear Proc. Geophys., 6,179 .

Kruer, W. L., J. M. Dawson, and B. Rosen (1973), The dipole expansion methods for plasma simulation, J. Comput. Phys., 13, 114.

Leboeuf, J. N., D. R. Thayer, R. D. Sydora, and P. H. Diamond (1986), Particle simulations of current-driven drift waves in shearless and sheared magnetic fields, Phys. Fluids, 29, 4147.

Lee, W. W., and H. Okuda (1978), A simulation model for studying lowfrequency microinstabilities, J. Comput. Phys., 26, 139.

Lyons, L. R., and T. W. Speiser (1982), Evidence for current sheet acceleration in the geomagnetic tail, J. Geophys. Res., 87, 2276.

Lysak, R. L., M. K. Hudson, and M. Temerin (1980), Ion heating by strong electrostatic ion cyclotron turbulence, J. Geophys. Res., 85, 678

Matsumoto, H., H. Kojima, T. Miyatake, Y. Omura, M. Okada, I. Nagano, and M. Tsutui (1994), Electrostatic solitary waves (ESW) in the magnetotail: BEN waveforms observed by GEOTAIL, Geophys. Res. Lett., 21, 2915

Nishikawa, K.-I., L. A. Frank, and C. Y. Huang (1988), Simulation of electrostatic turbulence in the plasma sheet boundary layer with electron currents and bean-shaped ion beams, J. Geophys. Res., 93, 5929.

Olsson, A., P. Janhunen, and W. K. Peterson (2004), Ion shell distributions as free energy source for plasma waves on auroral field lines mapping to plasma sheet boundary layer, Ann. Geophys., 22, 2115.

Omura, Y., H. Matsumoto, T. Miyake, and H. Kojima (1996), Electron beam instabilities as generation mechanism of electrostatic solitary waves in the magnetotail, J. Geophys. Res., 101, 2685.

Persson, H. (1963), Electric field along a magnetic line of force in a low density plasma, Phys. Fluids, 6, 1756.

Rème, H., et al. (2001), First multispacecraft ion measurements in and near the Earth's magnetosphere with the identical Cluster ion spectrometry (CIS) experiment, Ann. Geophys., 19, 1303.

Scarf, F. L., L. A. Frank, K. Ackerson, and R. P. Lepping (1974), Plasma wave turbulence at distant crossings of the plasma sheet boundaries and neutral sheet, Geophys. Res. Lett., 1, 189.

Schriver, D., and M. Ashour-Abdalla (1987), Generation of high frequency broadband electrostatic noise: The role of cold electrons, J. Geophys. Res., 92, 5087.

Schriver, D., and M. Ashour-Abdalla (1990), Cold plasma heating in the plasma sheet boundary layer: Theory and simulations, J. Geophys. Res., 95, 3987

Schriver, D., M. Ashour-Abdalla, and R. L. Richard (2001), Formation of electrostatic potential drops in the auroral zone, Phys. Chem. Earth, Part $C, 26,65$.

Sentman, D. D., J. N. Leboeuf, T. Katsouleas, R. W. Huff, and J. M. Dawson (1986), Electrostatic instabilities of velocity space shell distributions in magnetized plasmas, Phys. Fluids, 29, 2569.

Sentman, D. D., J. N. Leboeuf, T. Katsouleas, R. W. Huff, and J. M. Dawson (1987), Erratum: 'Electrostatic instabilities of velocity space shell distributions in magnetized plasmas', Phys. Fluids, 30, 1575.

Shevchenko, V., V. Galinsky, R. Sagdeev, and D. Winske (2004), Macroscale instability of ion shell distribution function in the divergent solar wind, Phys. Plasmas, 11, 4290

Sydora, R. D., J. N. Leboeuf, and T. Tajima (1986), Two- and three-dimensional particle simulation models for study of plasma microinstabilities, Supercomputers, edited by F. W. Matsen and T. Tajima, p. 366, Univ. of Texas Press, Austin. 
Tataronis, J. A., and F. W. Crawford (1970a), Cyclotron harmonic wave propagation and instabilities, I. Perpendicular propagation, J. Plasma Phys., 4, 231

Tataronis, J. A., and F. W. Crawford (1970b), Cyclotron harmonic wave propagation and instabilities, II. Oblique propagation, J. Plasma Phys., 4, 249.

Wygant, J. R., et al. (2000), Polar spacecraft based comparisons of intense electric fields and Poynting flux near and within the plasma sheet-tail lobe boundary to UVI images: An energy source for the aurora, J. Geophys. Res., 105, 18,675.

Zelenyi, L. M., R. A. Kovrashkin, and J. M. Bosqued (1990), Velocitydispersed ion beams in the nightside aurora zone: AUREOL 3 observations, J. Geophys. Res., 95, 12,119.

M. Ashour-Abdalla and D. Schriver, Institute of Geophysics and Planetary Physics, University of California, Los Angeles, CA 90095, USA. (mabdalla@igpp.ucla.edu)
J.-M. Bosqued, CESR, CNRS, 9, Avenue Colonel Roche, BP 4346, F-31028 Toulouse, France.

N. Cornilleau-Wehrlin, CETP, IPSL, 10-12, avenue de l'Europe, F-78140 Velizy, France.

A. N. Fazakerley and A. Marchaudon, Mullard Space Science Laboratory, University College London, Holmbury St. Mary, Dorking RH5 6NT, UK.

J. N. Leboeuf, Department of Physics, University of California, Los Angeles, CA 90095, USA.

V. Sotnikov, Department of Physics, University of Nevada, Reno, NV 89557, USA. 